\title{
Linear wavelength correlation matrices of photospheric and chromospheric spectral lines
}

\section{Observations vs. modeling}

\author{
C. A. R. Beck ${ }^{1,2}$ and W. Rammacher ${ }^{3}$ \\ 1 Instituto de Astrofísica de Canarias (CSIC), Vía Lactéa, 38205 La Laguna, Tenerife, Spain \\ e-mail: cbeck@iac.es \\ 2 Departamento de Astrofísica, Universidad de La Laguna, 38205 La Laguna (Tenerife), Spain \\ 3 Kiepenheuer-Institut für Sonnenphysik, Schöneckstr. 6, 79104 Freiburg, Germany \\ e-mail: wr@kis.uni-freiburg.de
}

Received 18 March 2009 / Accepted 13 November 2009

\section{ABSTRACT}

\begin{abstract}
Context. The process that heats the solar chromosphere is a difficult target for observational studies because the assumption of local thermal equilibrium (LTE) is not valid in the upper solar atmosphere, which complicates the analysis of spectra.

Aims. We investigate the linear correlation coefficient between the intensities at different wavelengths in photospheric and chromospheric spectral lines because the correlation can be determined directly for any spectra from observations or modeling. Waves which propagate vertically through the stratified solar atmosphere affect different wavelengths at different times when the contribution functions for each wavelength peak in different layers. This leads to a characteristic pattern of (non-)coherence of the intensity at various wavelengths with respect to each other which carries information on the physical processes.

Methods. We derived the correlation matrices for several photospheric and chromospheric spectral lines from observations. We separated locations with a significant photospheric polarization signal and thus magnetic fields from those without a polarization signal. For comparison with the observations, we calculated correlation matrices for spectra from simplified LTE modeling approaches, 1-D NLTE simulations, and a 3-D MHD simulation run. We applied the correlation method also to temperature maps at different optical depth layers derived from a LTE inversion of Ca II H spectra.

Results. We find that all photospheric spectral lines show a similar pattern: a pronounced asymmetry of the correlation between line core and red or blue wing. The pattern cannot be reproduced with a simulation of the granulation pattern, but with waves that travel upwards through the formation heights of the lines. The correct asymmetry between red and blue wing only appears when a temperature enhancement occurs simultaneously with a downflow velocity in the wave simulation. All chromospheric spectral lines show a more complex pattern. The 1-D NLTE simulations of monochromatic waves produce a correlation matrix that qualitatively matches the observations near the very core of the $\mathrm{Ca}$ II $\mathrm{H}$ line. The photospheric signature is well reproduced in the correlation matrix derived from the 3-D MHD simulation.

Conclusions. The correlation matrices of observed photospheric and chromospheric spectral lines are highly structured with characteristic and different patterns in every spectral line. The comparison with matrices derived from simulations and simple modeling suggests that the main driver of the detected patterns are upwards propagating waves. Application of the correlation method to 3-D temperature cubes seems to be a promising tool for a detailed comparison of simulation results and observations in future studies.
\end{abstract}

Key words. Sun: chromosphere - Sun: oscillations

\section{Introduction}

The Ca II H and K spectral lines have been one of the most important sources of chromospheric diagnostics in solar and stellar physics. The lines show a generally complex behavior with some shared characteristics. Both $\mathrm{H}$ and $\mathrm{K}$ exhibit sudden "bright grains" (BGs) where wavelengths in and near the line core revert to emission for a short duration (e.g., Rutten \& Uitenbroek 1991). The BGs are often repetitive with a cadence of around 180-200 s (e.g., Beck et al. 2008, and references therein). As an explanation for the BGs the (upwards) propagation of acoustic waves has been suggested soon after their first detection, because the BGs can often be seen to appear in the line wings already about 50-100 sec before the emission in the core (see Fig. 1, or Liu 1974; Beck et al. 2008), which supports the idea of a wave propagation.

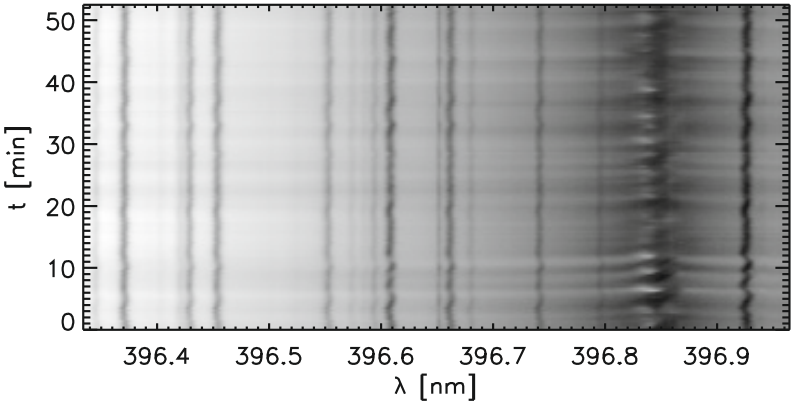

Fig. 1. Example of observed Ca II H spectra.

Due to their formation above the photosphere in a layer with mostly NLTE conditions, the theoretical treatment and the 
interpretation of the $\mathrm{Ca}$ line behavior in observations is difficult. The assumption of LTE breaks down for the formation of the line cores of the Ca II resonance lines because of the low gas density, but is still valid in the wings of these lines. For the analysis of the spectra exist fortunately also diagnostic methods that are independent of the LTE or non-LTE assumption. One of these methods is the matrix of the linear correlation coefficient of the intensity at different wavelengths. The correlation shows a characteristic pattern if there is a causal relationship between different atmospheric layers. When a time lag is introduced in the analysis of a temporal sequence of spectra, the pattern changes accordingly, as an intensity variation at a wavelength $\lambda_{1}$ at a time $t_{1}$ appears at a different time $t_{2}$ in $\lambda_{2}$. One of the great advantages of the correlation coefficient is that it can be calculated for observed or synthetic spectra from either numerical or analytical models in the same way for any kind of spectral line or also continuum levels. Interestingly, the correlation matrices can also be derived for other physical quantities, like temperature cuts at geometrical heights in simulations, which widens the field of possible applications of the analysis method.

Since there is no current literature on the theoretical expectations or the interpretation of the correlation matrices (but see for example Cram (1978) for a similar type of study in the Fourier domain), we want to present in this contribution only some examples of correlation matrices for observations and four numerical experiments with a different degree of sophistication. The equation for calculating the correlation matrices is explained in Sect. 2. We show the correlation matrices for observations of spectral lines from near-UV (396 nm) to near-IR $(1.5 \mu \mathrm{m})$, covering several photospheric and chromospheric spectral lines, in Sect. 3. The corresponding results for the numerical experiments are shown in Sect. 4. Section 5 shows correlation matrices for temperature as an example of another physical quantity to which the method can be applied. We summarize and discuss the findings in Sect. 6, whereas Sect. 7 gives any conclusions that can be drawn at this time.

\section{Calculation of correlation matrices}

We use the standard definition for the linear correlation coefficient $r_{A B}$ for two quantities $A$ and $B$ :

$$
r_{A B}=\frac{1}{\sqrt{\int_{V} A^{\prime 2} \cdot \int_{V} B^{\prime 2}}} \int_{V} A^{\prime} \cdot B^{\prime},
$$

where the integration $\int_{V}$ is to be executed over all elements of $A$ and $B$, respectively. $A^{\prime}$ stands for $A-\langle A\rangle$ where \langle\rangle denotes the average.

For our purpose of wavelength correlations, we normally used a monochromatic image in one wavelength, $I\left(\lambda_{1}\right)$, as 2-D image $A$, to be correlated with $B=I\left(\lambda_{2}\right)$ to obtain $r_{\lambda_{1} \lambda_{2}}$. We also selected in some cases subfields of observations or simulations to investigate the influence of magnetic fields on the correlation matrices. The correlation matrices which relate spectra inside the same spectral range are square and symmetric to the diagonal; i.e., $r_{\lambda_{1} \lambda_{2}}=r_{\lambda_{2} \lambda_{1}}$ (see for example Fig. 2). For crosscorrelations of different spectral ranges, the resulting matrix is in general rectangular without a symmetry axis. Cross-correlation matrices between different spectral ranges are especially interesting where time-series of spectra are available, because they allow the study of the variation of the correlation for different time lags. For propagating waves and a finite difference in formation height, the maximum correlation should be reached for a time lag corresponding to the wave travel time between the two height layers. Cross-correlations and correlations with a time lag will be investigated in a subsequent publication.

In the present case, we used both large-area scans yielding monochromatic images $I(\lambda, x, y)$ with the spatial coordinates $(x, y)$, and spatio-temporal time series giving $I(\lambda, x, t)$. Equation (1) is insensitive to this difference. We note that $I(\lambda, x, y)$ and $I(\lambda, x, t)$ are equivalent only in a statistical sense if they both cover a sufficient large number of periods, or more general, variation times. In a time series, all phases of the variations are seen at a fixed location, whereas for a large area map the phases are sampled at different locations in a random state. This requires the spatial maps to be as large as possible to avoid contamination of the correlation matrices by a coherent evolution in small subfields $\left(\gg 5^{\prime \prime}\right)$ and the time-series to cover at least some variation cycles ( $\gg 5 \mathrm{~min})$.

\section{Correlation matrices from observations}

To obtain correlation matrices for the various spectral lines, we chose several observations of quiet Sun (QS) areas at disk center taken between 2006 and 2009. The QS data were taken with slitspectrograph systems, where the 1-D slit is stepped across the solar image to obtain a 2-D field of view (FOV). Different spatial locations in the scanning direction are thus sampled at different times. The observation data were acquired with the spectropolarimeters POLIS (Beck et al. 2005, $396 \mathrm{~nm}, 630 \mathrm{~nm}$ ), TIP (Collados et al. 2007, $1083 \mathrm{~nm}, 1565 \mathrm{~nm}$ ), and a spectroscopic setup using the main spectrograph of the German Vacuum Tower Telescope (VTT) for Ca II IR $866 \mathrm{~nm}$. Simultaneously with the Ca II IR 866 nm line, Ca II H intensity spectra were also recorded for the investigation of cross-correlations; these spectra were, however, not used here. For Ca II IR 854 nm, we have two data sets. The first was recorded at the VTT with a similar setup as for Ca II IR $866 \mathrm{~nm}$. The second data set of Ca II IR $854 \mathrm{~nm}$ was taken with the IBIS spectrometer (Cavallini 2006) of the Dunn Solar Telescope (Sac Peak/NSO) in spectropolarimetric mode. IBIS is a Fabry-Perot-Interferometer based 2-D spectrometer. We selected observations that covered large FOVs on or near disk center and one time-series; the Ca II IR $854 \mathrm{~nm}$ observation with IBIS is the only exemption (no QS, but a pore off the disk center). Appendix A shows the observed FOVs; more details on each observation are given in Table A.1.

POLIS, TIP, and IBIS measured the Stokes vector in magnetic sensitive spectral lines, which additionally gives the option to localize magnetic fields and to avoid or include the respective positions in the analysis. To investigate the influence of photospheric magnetic fields on the correlation matrices, we created masks of magnetic field locations for each data set. We integrated the absolute circular polarization signal $|V|$ in wavelength and then set a variable threshold depending on the integration time and the magnetic sensitivity of the observed spectral lines. Locations with a polarization signal below (above) the threshold were assumed to be nearly field-free (magnetic) and are labeled "unpolarized/unpol." ("polarized/pol.") in the following. As a third sample, we also calculated the correlation matrix using the full FOV, which is better suited for comparisons when no mask of field locations can be made. Appendix A also shows the Stokes $V$ maps and the masks of each observation. For the observations of Ca II IR at $866 \mathrm{~nm}$, no polarimetric measurements were available. We did not try to define a mask of locations with presumably magnetic fields from the line core intensity map (see Fig. A.3), but only calculated the correlation matrix of the full FOV in that case. For the observations of Ca II IR at $854 \mathrm{~nm}$ 

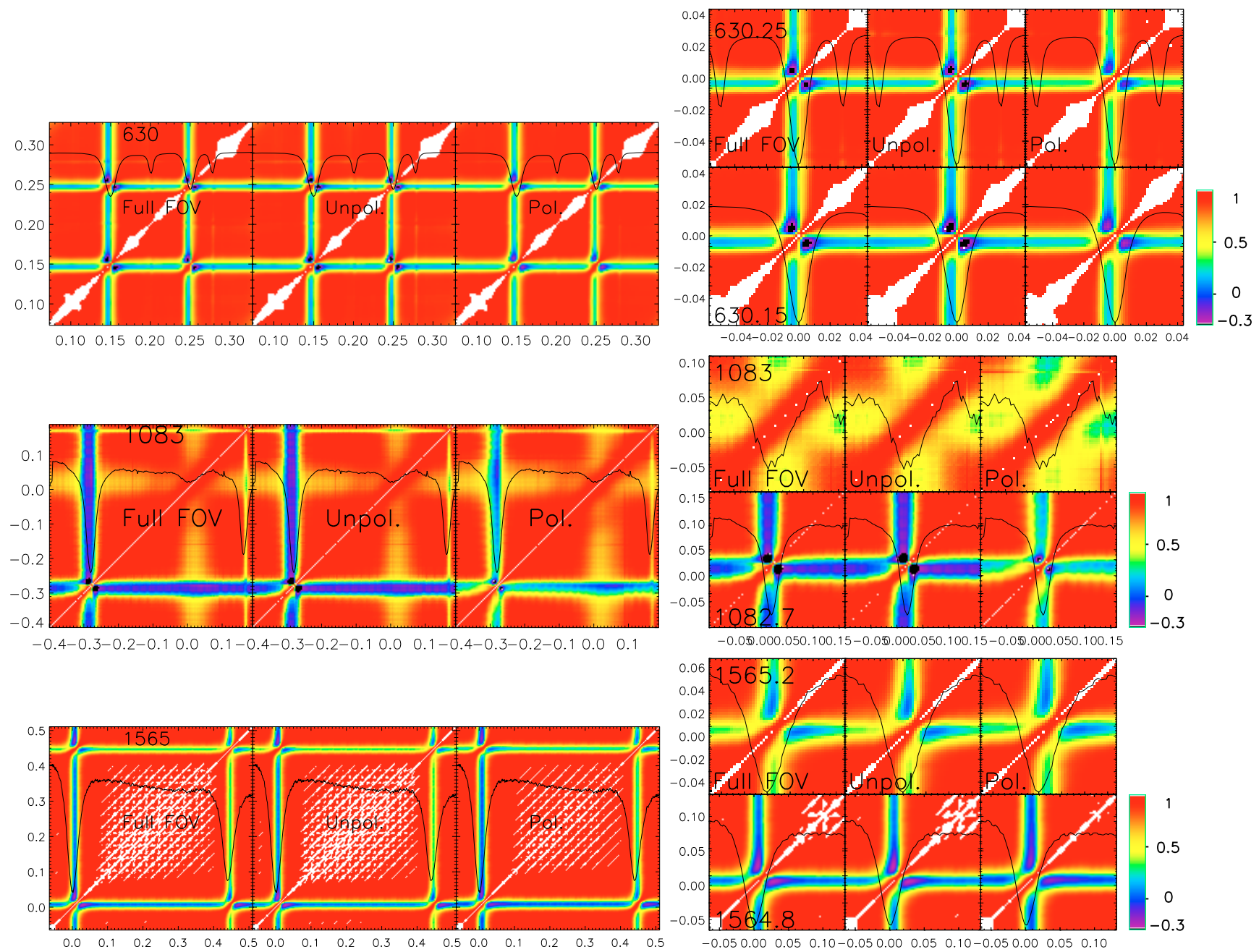

Fig. 2. Correlation matrices of the $630 \mathrm{~nm}, 1083 \mathrm{~nm}$ and $1565 \mathrm{~nm}$ range. The correlation matrix shows the linear correlation coefficient $r_{\lambda_{i} \lambda}$ between the intensities at the wavelengths $\lambda_{i}$ (along $x$ ) and $\lambda_{j}$ (along $y$ ). The correlation value is color-coded according to the color bars at the right. Left: full observed wavelength range. Right: magnification of line-core wavelengths. The matrices of the three samples made from the FOV (full FOV, unpol(arized) and pol(arized)) are aligned left to right in each plot. A line profile is overplotted as a black line. White color corresponds to a correlation value of 1. Top and bottom row of the magnification show $630.25 \mathrm{~nm}$ and $630.15 \mathrm{~nm}$ (630), He I $1083 \mathrm{~nm}$ and Si I $1082.7 \mathrm{~nm}$ (1083), and $1565.2 \mathrm{~nm}$ and $1564.8 \mathrm{~nm}$ (1565), respectively. The wavelengths for the left graphs are $\lambda-630 \mathrm{~nm}, \lambda-1083 \mathrm{~nm}$ and $\lambda-1564.85 \mathrm{~nm}$, respectively; for the right graphs, wavelengths in $\mathrm{nm}$ are relative to the rest wavelength of the line shown.

taken at the VTT in 2009, we have not yet aligned the data to the simultaneous polarimetric observations with TIP and POLIS, thus there is no mask available either.

Figures 2-4 show the correlation matrices, either obtained by averaging the matrices of several observations in each wavelength range whenever multiple suited data sets were available, or derived from individual observations (Ca II IR lines). Each figure shows the correlation matrix for the full spectral range covered in each observation, and additionally a magnified view of individual spectral lines for the TIP and POLIS data sets. Besides for Ca II IR $866 \mathrm{~nm}$ and the QS data set of Ca II IR $854 \mathrm{~nm}$, each time the three samples "full FOV", "unpolarized" and "polarized" are shown left to right in each plot; for Ca II IR $866 \mathrm{~nm}$ and the QS data set of Ca II IR $854 \mathrm{~nm}$, only the matrix of the full FOV is shown (top and middle panel of Fig. 4). We did not find significant changes of the correlation matrices with different integration times for photospheric lines, even if for example integration times between 5 and $30 \mathrm{~s}$ were used for $1565 \mathrm{~nm}$ in the various observations (cf. Table A.1). The spectra at $1.56 \mathrm{mi}-$ cron taken with the TIP II camera (Collados et al. 2007) have a pattern of spectral fringes (see, e.g., Beck \& Rezaei 2009) which produces high correlation on stripes parallel to the diagonal that are not of solar origin. We first calculated correlation matrices for a time-series with a fixed slit separately, but they were qualitatively identical to those from large-area maps and thus were simply included in the averaging. The He I $1083 \mathrm{~nm}$ line shows nearly no absorption in QS conditions and thus yields little structure, but has been displayed for completeness.

The correlation matrices for the intensity at various wavelengths and spectral lines forming in photospheric atmospheric layers (continuum intensity, 630.15 and $630.25 \mathrm{~nm}$, Si I $1082.7 \mathrm{~nm}, 1564.8$ and $1565.2 \mathrm{~nm}$, the line blends in the wing of $\mathrm{Ca}$ II $\mathrm{H}$ ) are very similar regardless of wavelength in the near-UV, visible or near-IR. Correlations between the intensities at continuum wavelengths are always close to 1 . Photospheric spectral lines show a reduced correlation up to anti-correlation if the intensities at wavelengths in or close to the line core are correlated with the intensities at continuum wavelengths. Wavelengths in the red and blue wing are not equivalent: the reduction of the correlation between the core and the red wing is always stronger than for the blue wing. The absolute value of the correlation is smaller for $1565.2 \mathrm{~nm}$ and $630.25 \mathrm{~nm}$ than for 

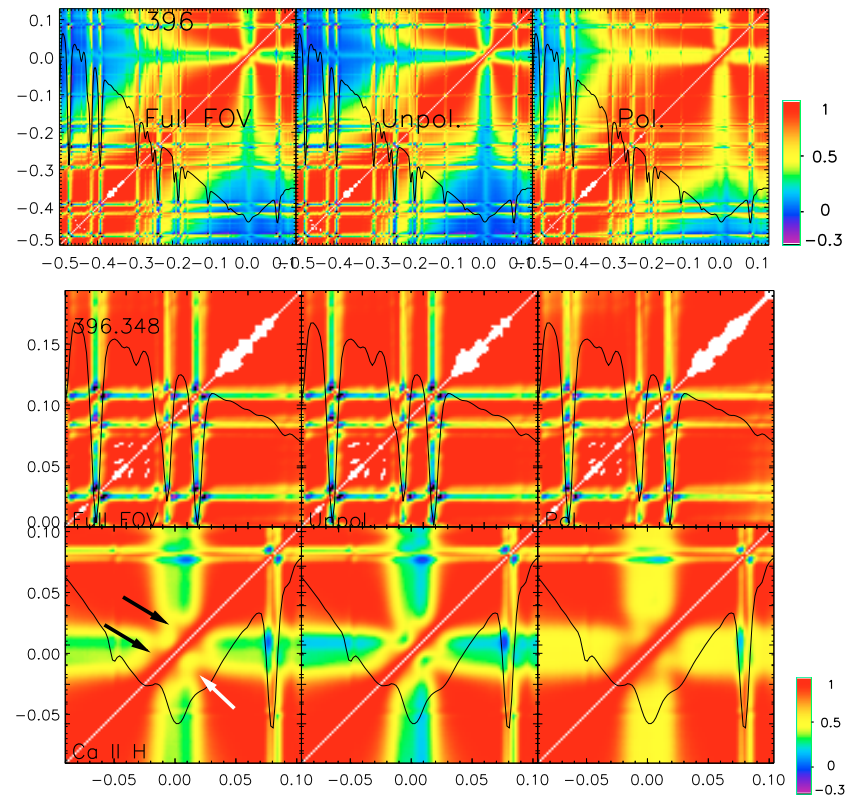

Fig. 3. Same as Fig. 2 for Ca II H at 396 nm. Bottom graph: magnification of line cores of some blends ( $\mathrm{Fe}$ I at $396.455 \mathrm{~nm}$, Ti I at $396.427 \mathrm{~nm}$, and CrI at $396.369 \mathrm{~nm}$ ) (top) and Ca II H $396.85 \mathrm{~nm}$ (bottom). Wavelengths are $\lambda-396.85 \mathrm{~nm}$ for all plots besides the middle row, where the wavelength is given as $\lambda-396.34 \mathrm{~nm}$.

$1564.8 \mathrm{~nm}$ or $630.15 \mathrm{~nm}$. We ascribe this fact to the line depth of the respective spectral line.

The correlation of the line-core intensity of a photospheric line with the intensity at close-by wavelengths $\left(r_{\lambda_{1} \lambda_{1} \pm \Delta \lambda}\right)$ gives roughly the same pattern as the correlation with the intensity near another line core $\left(r_{\lambda_{1} \lambda_{2} \pm \Delta \lambda}\right)$ each time $\lambda_{2}$ corresponds to a photospheric line-core wavelength, doubling the pattern of the stripes of reduced correlation for each spectral line present inside the wavelength range (Fig. 2). The correlation changes only slightly from line to line, which is due to the fact that the respective photospheric line pairs in the visible and near-IR wavelength ranges $(1564.8 / 1565.2 \mathrm{~nm}, 630.15 / 630.25 \mathrm{~nm})$ have similar formation heights (Cabrera Solana et al. 2005). If the formation height differs strongly, like for Si I $1082.7 \mathrm{n}$ and He I $1083 \mathrm{~nm}$ or the line core and all the line blends of $\mathrm{CaII} \mathrm{H}, r_{\lambda_{1} \lambda_{1} \pm \Delta \lambda}$ and $r_{\lambda_{1} \lambda_{2} \pm \Delta \lambda}$ show little similarity.

With respect to the different samples made in each FOV (full FOV, unpol., pol.) only small changes are seen. A clear trend is that for the polarized sample the width of the stripes with reduced correlation related to the line cores is slightly larger, clearly seen only for $630.25 \mathrm{~nm}$ and $1564.8 \mathrm{~nm}$. This is due to the splitting of the lines inside magnetic fields, leading to multiple spectral components in the intensity profile ${ }^{1}$. The correlation values between line-core intensities and the intensities at continuum wavelengths are always higher in the polarized sample than in the unpolarized sample; this is best seen for Ca II $\mathrm{H}$ and Si I 1082.7 nm where the graphs of "unpol" and "pol" are markedly different in the value of the correlation coefficient. The higher correlation could be due to the field-strength dependent shift of the optical depth scale. The intensity will be enhanced at all wavelengths on locations with magnetic fields, which itself will not increase the correlation due to the subtraction of the average values in Eq. (1). But if a dependence of the intensity enhancement on the field strength exists as well, $I\left(\lambda_{i}, x, y\right)$ and

1 Tested on synthetic spectra.

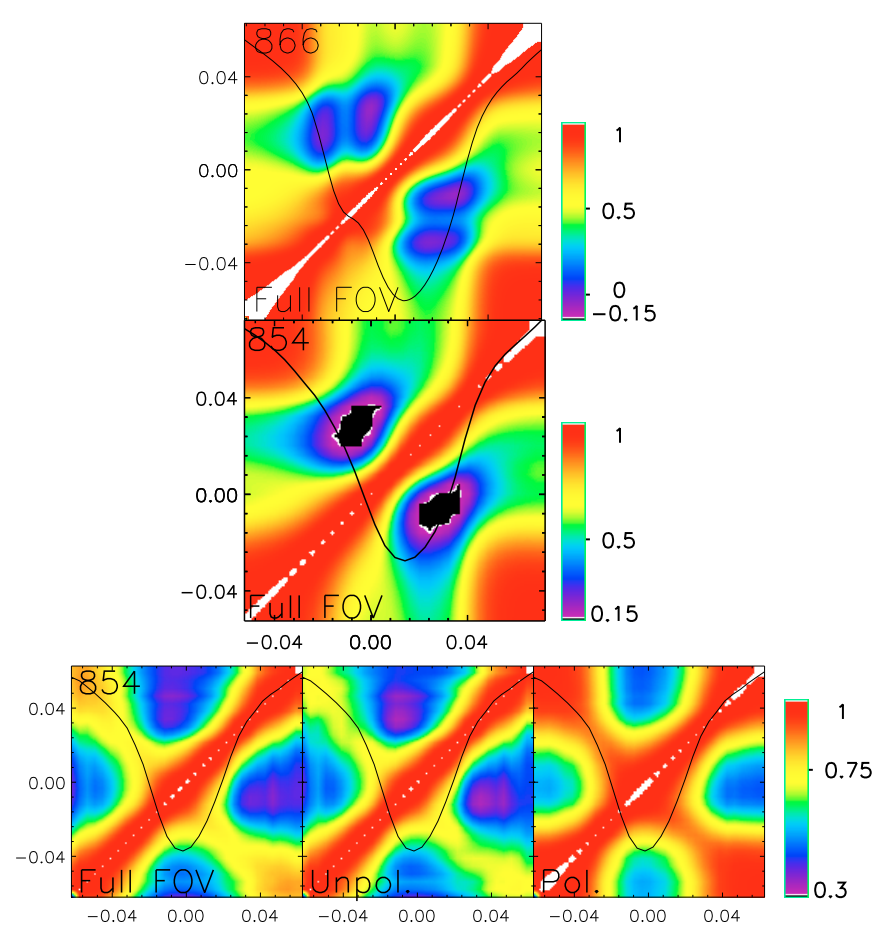

Fig. 4. Top: correlation matrix for Ca II IR $866 \mathrm{~nm}$ for the full FOV. Wavelengths are $\lambda-866.215 \mathrm{~nm}$. Middle: correlation matrix for Ca II IR $854.2 \mathrm{~nm}$ for the full FOV (slit spectrograph data, disk center). Bottom: correlation matrices for Ca II IR $854.2 \mathrm{~nm}$ (IBIS data, off center in an active region). Wavelengths are $\lambda-854.215 \mathrm{~nm}$.

$I\left(\lambda_{j}, x, y\right)$ will scale in the same way. For example, for all locations $(x, y)$ with stronger (weaker) than average magnetic fields, $\mathrm{d} I_{i}=I\left(\lambda_{i}, x, y\right)-\left\langle I\left(\lambda_{i}\right)\right\rangle$ and $\mathrm{d} I_{j}=I\left(\lambda_{j}, x, y\right)-\left\langle I\left(\lambda_{j}\right)\right\rangle$ will be greater (smaller) than zero at the same time, increasing the fraction of locations with a positive correlation $\left(\mathrm{d} I_{i} \cdot \mathrm{d} I_{j}>0\right)$.

The structures in the correlation matrices of all chromospheric Ca II lines are significantly different from the photospheric cases. For Ca II H (Fig. 3), the correlation of the intensities at wavelengths in or close to the $\mathrm{Ca}$ line core with that at wavelengths separated by around $0.3 \mathrm{~nm}$ gives a reduced correlation over an extended wavelength range; the reduction of the correlation is not restricted only to a small stripe near the line core like for the photospheric lines. Around the diagonal, a band of high correlation exists, whose width changes with wavelength, increasing from the $\mathrm{Ca}$ II $\mathrm{H}$ line core up to a $0.2 \mathrm{~nm}$ wavelength separation. Between $0.2 \mathrm{~nm}$ and $0.3 \mathrm{~nm}$ there is decrease of the width, which then changes to an increase again. The effect is most pronounced for the polarized case, where the high correlation extends over almost the full wavelength range for wavelengths outside of spectral lines.

The photospheric line blends in the $\mathrm{Ca}$ line wing show the same pattern with the red/blue asymmetry near their line core as the other photospheric lines, but the $\mathrm{Ca}$ II $\mathrm{H}$ line core shows a pattern which is more strongly structured. It consists of two stripes of reduced correlation starting from the line core as for the photospheric lines, but at around $0.15 \mathrm{~nm}$ from the line core a small area of reduced correlation in the shape of a half-circle is visible which is missing for the other lines (white arrow in Fig. 3). The feature connects the two stripes of reduced correlation going to red and blue wing, respectively. Centered on around $\pm 0.02 \mathrm{~nm}$ from the line core, two small squares of high correlation can be seen (black arrows in Fig. 3), directly above (left) of the lower half-circle. The blue/red asymmetry of the 


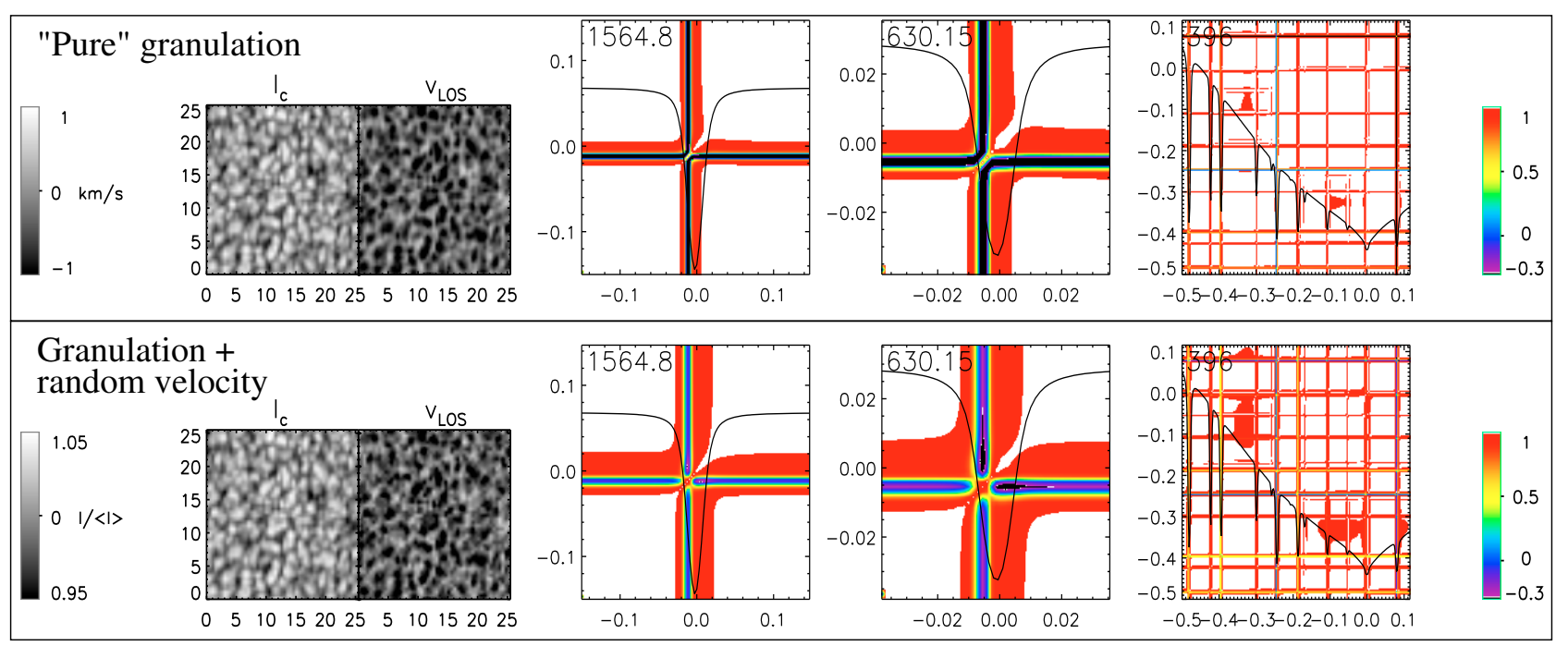

Fig. 5. Simulation of granulation and resulting correlation matrices. Top row: velocity directly proportional to $I_{\mathrm{c}}$. Bottom row: same as before, but with a random velocity added. 1st column: continuum intensity $I_{\mathrm{c}}$ map at left and LOS line-core velocity map at right. Tick marks are in arcsec, intensity and velocity color bars are displayed in the bottom and top row, respectively. 2nd to 4th column: correlation matrices for $1564.8 \mathrm{~nm}$, $630.15 \mathrm{~nm}$ and the full Calcium line profile. Wavelengths are given in $\mathrm{nm}$ relative to the rest wavelength of each line.

photospheric lines is missing completely, both wings seem to be equivalent for the Ca II $\mathrm{H}$ line core. Ca II IR at $866 \mathrm{~nm}$ (top panel of Fig. 4) also shows a more complex pattern compared to photospheric lines. It exhibits two patches of reduced correlation at around $+0.03 \mathrm{~nm}$ from the core, presumably caused by a strong Fe I line blend at this wavelength, but the blue and red wing are equivalent without asymmetry ${ }^{2}$. The correlation matrix for the QS data of Ca II IR at $854 \mathrm{~nm}$ (middle panel of Fig. 4) is fairly similar to that of the $866 \mathrm{~nm}$ line, but shows only a single patch of reduced correlation. The structure seen in the off-center observations of Ca II IR at $854 \mathrm{~nm}$ is quite different. The stripes of reduced correlation to the red and blue of the line core do not connect directly like in the QS data; especially for the polarized sample a high correlation is found between the branches from the core to blue and red. We suggest that the strong variation of the correlation matrix for one and the same spectral line is due to both the solar structure of the observed FOV (cf. Fig. A.3) and to the off-center position, similar to the differences in the temperature correlations later on in Sect. 5. In total, the photospheric lines present a simple structure in the correlation matrices dominated by the red/blue asymmetry, whereas all chromospheric lines show a more detailed fine-structure.

\section{Numerical and (semi-)analytical spectra}

For comparison to the observed correlation matrices, we used spectra obtained from four approaches differing in the sophistication of the method used for the generation of the spectra.

\subsection{Granulation simulation}

The convective energy transport and the resulting granulation pattern dominates the spatial distribution of the continuum intensity in the solar photosphere, and also all layers up to around $300-400 \mathrm{~km}$ above $\log \tau_{500 \mathrm{~nm}}=0$ that are relevant for the formation of photospheric spectral lines. To estimate the contribution of granulation to the wavelength correlation matrices, we created a synthetic data set that includes only granulation and no

\footnotetext{
${ }^{2}$ Only the wavelength range near the line core is shown in Fig. 4.
}

waves of any kind. We used a subsection of the continuum intensity map of a long-exposed QS observation on the disk center obtained with the TIP instrument at 1.56 micron (Beck \& Rezaei 2009) to define the intensity pattern in a $25 \operatorname{arcs}^{2}$ area (see left column of Fig. 5). We defined the LOS velocity to be inverse proportional to the intensity by

$v_{\mathrm{LOS}}(x, y)=-\Delta I_{\mathrm{c}}(x, y) \cdot 20.000 \mathrm{~ms}^{-1}$,

which yields velocities in around $\mathrm{a} \pm 1 \mathrm{~km} \mathrm{~s}^{-1}$ range, since $\Delta I_{\mathrm{c}}(x, y)=\left(I_{\mathrm{c}}(x, y)-\left\langle I_{\mathrm{c}}\right\rangle\right)$ lies between around 0.95 and 1.05 for the near-IR observations.

The temperature stratification was defined using a modified version of the Harvard Smithsonian Reference Atmosphere model (HSRA, Gingerich et al. 1971). We used an optical depth range from $\log \tau=1.4$ to $\log \tau=-6$. Up to $\log \tau=-4$, we used the values of the original HRSA model, but in the layers above we substituted the chromospheric temperature rise in the HSRA with the corresponding values of the Holweger-Mueller model (HOLMUL, Holweger \& Mueller 1974). The HOLMUL model gives to first order an atmosphere in radiative equilibrium without a chromospheric temperature rise. The main motivation for this choice was that otherwise Ca spectra with (unobserved) large single-peaked emission in the line core result in an LTE synthesis, but Rezaei et al. (2008) also showed that the solar chromosphere partially reverts to such a low-energy state in the absence of shock waves. The temperature stratifications of the two models were smoothly connected by adding the difference $\Delta T_{\log \tau=-4}=T(\text { HSRA })_{\log \tau=-4}-T(\text { HOLMUL })_{\log \tau=-4}$ to the values of the HOLMUL model. The gas and electron densities above $\log \tau=-4$ were derived by an extrapolation of the exponential decrease of the HSRA model below $\log \tau=-4$ to the rest of the optical depth range. The atmosphere was not put to hydrostatic equilibrium, as only a qualitative guess was desired.

For the granulation simulation, the temperature on each point $(x, y)$ was additionally modified by multiplying the temperature stratification with $1+\Delta I_{\mathrm{c}}(x, y)$, yielding a variation of about $\pm 300 \mathrm{~K}$ at continuum forming layers. Given temperature stratification and LOS velocity, we synthesized spectra for the $1.56 \mathrm{mi}-$ cron range, $630 \mathrm{~nm}$ range, and $\mathrm{Ca} \mathrm{II} \mathrm{H}$, respectively, in LTE with the SIR code (Ruiz Cobo \& del Toro Iniesta 1992). The resulting 


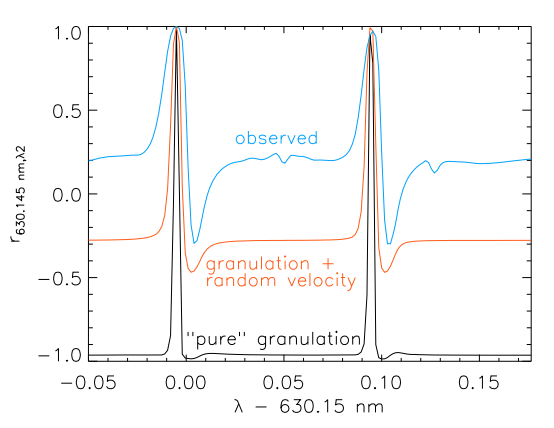

Fig. 6. Cuts through the correlation matrices at a wavelength of $630.145 \mathrm{~nm}$. Black: "pure" granulation simulation $\left(v \propto I_{\mathrm{c}}\right)$. Red: same with added random velocity. Blue: observed correlation matrix.

correlation matrices for $1564.8 \mathrm{~nm}, 630.15 \mathrm{~nm}$, and the Ca II H line are shown in the top row of Fig. 5 from left to right. They show stripes of, in this case, not only reduced but anti-correlation starting at the line cores as in the observations, but do not exhibit any clear red/blue asymmetry when the velocity is directly proportional to the intensity (top row). The correlation values are much lower than for the observations and reach down to around -0.9 near the very line core of any photospheric line. For Ca II H, the correlation is basically unity over the complete wavelength range outside the photospheric line blends. The asymmetry for photospheric lines thus does not originate from the presence of hot upflows and cold downflows, if a perfect linear relation between intensity and velocity is assumed.

As a second test we used the same setup as above for the temperature and velocity, but added a random Gaussian variation of $150 \mathrm{~ms}^{-1}$ variance to the velocities derived from Eq. (2) and again calculated the correlation matrices from the corresponding synthetic spectra (bottom row in Fig. 5). The correlation matrices then show the a red/blue asymmetry for the photospheric lines, but the correlation values are still much lower than for the observations. The matrix of $\mathrm{Ca}$ II $\mathrm{H}$ does not change noticeably and has no resemblance to the observed correlation matrix in both cases. This is presumably also due to the change of temperature at all height layers by a constant factor to mimic the spatial distribution of the continuum intensity. The chromospheric temperature and hence the intensity at wavelengths forming in chromospheric layers is basically decoupled from the photospheric pattern (e.g., bottom panel of Fig. A.1, or Beck et al. 2009).

Figure 6 shows cuts through the correlation matrices at a wavelength of $630.145 \mathrm{~nm}$. It highlights the difference between the two granulation simulations with respect to the red/blue asymmetry: the "pure" granulation simulation has only a very small asymmetry with a slightly stronger anti-correlation to the red (black line with small dips near $0 \mathrm{~nm}$ and $0.10 \mathrm{~nm}$ ), which is significantly more pronounced in the simulation with the additional random velocity (red line). Even with the added velocity variation, the asymmetry still falls short of the observed behavior (blue line).

Figure 7 compares the velocities of the two synthetic approaches with the actually observed line-core velocity of the $1564.8 \mathrm{~nm}$ line in a scatterplot versus the continuum intensity. The scatter of the observed velocity (blue) around the linear relationship (red) defined by Eq. (2) is about three times as big as the tested $150 \mathrm{~ms}^{-1}$ variance (black). This suggests that the asymmetry between red and blue wing for wavelengths near the line core of photospheric lines is not due to the granulation pattern of hot upflows and cold downflows, but to the deviation
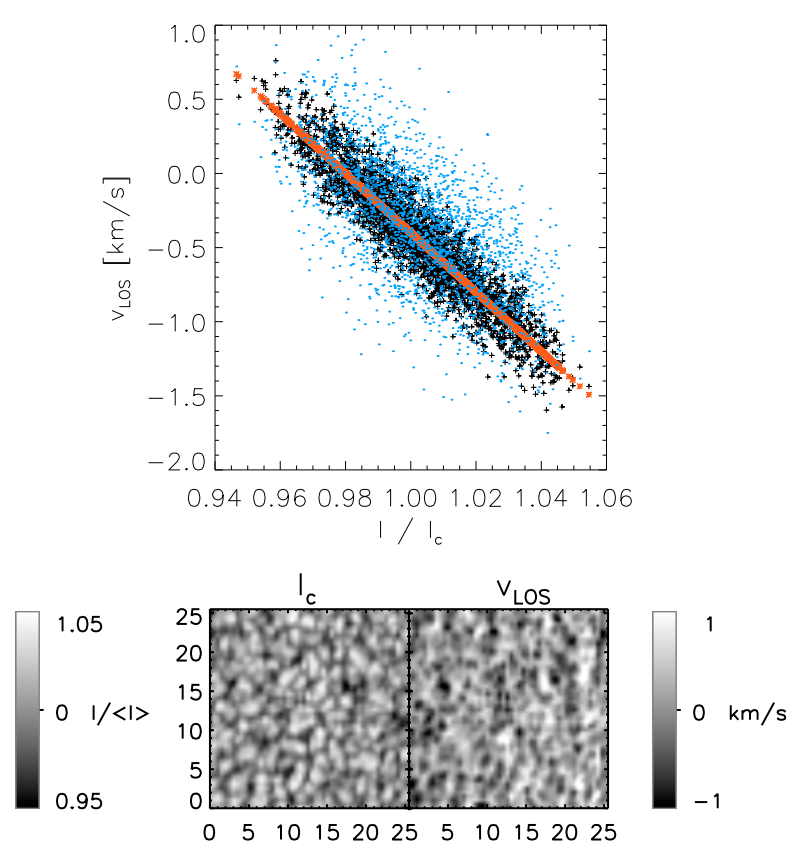

Fig. 7. Top: scatterplot of LOS line-core velocities vs continuum intensity $I_{\mathrm{c}}$. Red: velocity derived from Eq. (2). Black: velocity from Eq. (2) with random variation added. Blue: observed velocity. Bottom: comparison of observed intensity and LOS velocity. Tick marks are in arcsec.

from the purely convective source of the velocity field, i.e., the (magneto-)acoustic waves that usually have no strong signature in intensity at photospheric levels. The observed correlation matrix of the chromospheric Ca II $\mathrm{H}$ seems to be fully unrelated to a pure granulation pattern.

Another possible source of the asymmetry in the correlation matrix of photospheric lines could be the temperature (or intensity) contrast inversion between granules and intergranular lanes above the photosphere ("reverse granulation", see, e.g., Rutten et al. 2004). To find out whether this effect influences the correlation matrix, we investigated the relation between the continuum intensity $I_{\mathrm{c}}$ and the line-core intensity $I_{\text {core }}$ of the $1564.8 \mathrm{~nm}$ line. Figure 8 shows the corresponding maps for the full FOV of the long-exposed QS observations, together with a map of the polarization degree of the spectra. The line-core intensity was defined as the minimum intensity value in the spectral region of the $1564.8 \mathrm{~nm}$ line. The line-core intensity of the low-forming $1564.8 \mathrm{~nm}$ line (Cabrera Solana et al. 2005) still reflects the granulation pattern with a small positive correlation coefficient of 0.26 with respect to $I_{\mathrm{c}}$, i.e., $I_{\text {core }}$ is not yet influenced by the reverse granulation pattern. Mainly where the polarization degree is high, $I_{\text {core }}$ and $I_{\mathrm{c}}$ exhibit inverse patterns. The relation between $I_{\text {core }}$ and $I_{\mathrm{c}}$ shows a steeper slope for the granulation simulation than for the observations (bottom panel of Fig. 8), but this could be due to the stray-light contribution to the observed spectra. The binned values were derived like in Beck et al. (2007) by averaging the pairs $\left(I_{\mathrm{c}}, I_{\text {core }}\right)$ over bins in $I_{\mathrm{c}}$, where the plotted points correspond to $\left(\left\langle I_{\mathrm{c}}^{i}\right\rangle,\left\langle I_{\text {core }}^{i}\right\rangle\right)$. They correspond to the center of gravity of the $I_{\text {core }}$ distribution as a function of $I_{\mathrm{c}}$. From the positive correlation of $I_{\mathrm{c}}$ and $I_{\text {core }}$ and the correlation matrix of the granulation simulation, we think that we can exclude the (reverse) granulation pattern as a source of the asymmetry for photospheric lines, even if, e.g., the lines at $630 \mathrm{~nm}$ form in slightly higher layers closer to the reverse granulation pattern than the near-IR lines. 

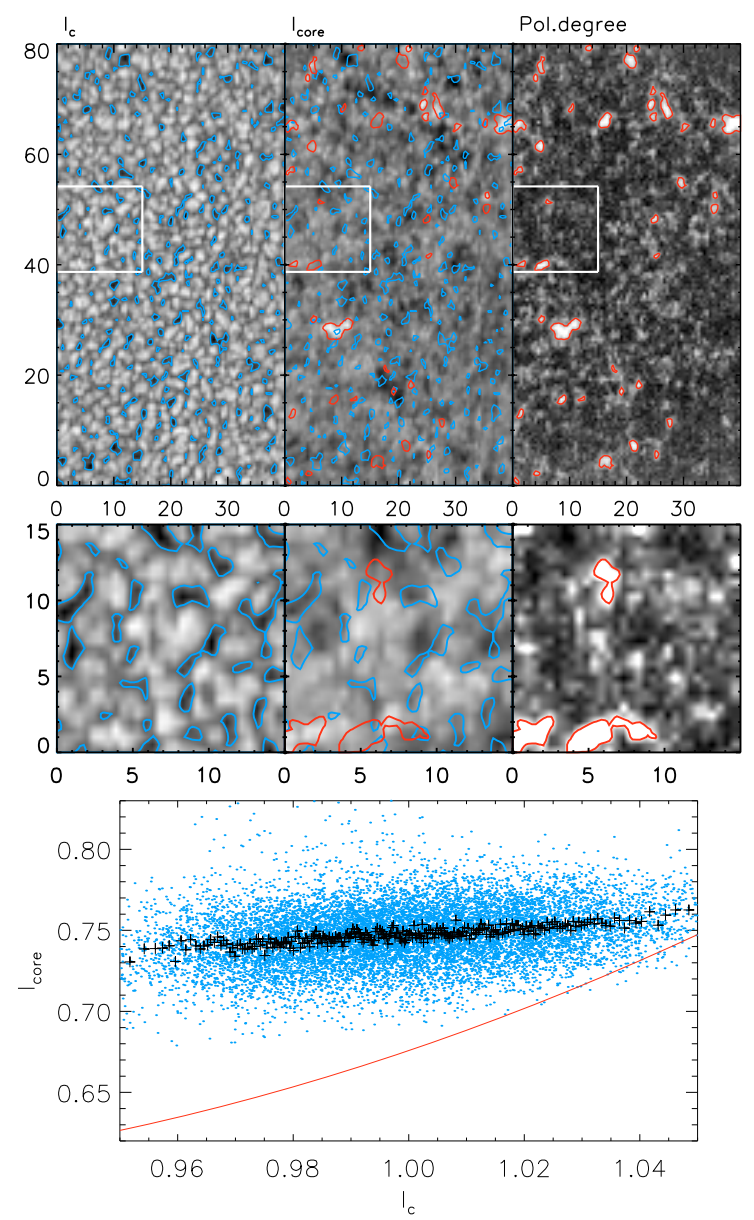

Fig. 8. Relation between continuum intensity $\left(I_{\mathrm{c}}\right)$ and line core intensity $\left(I_{\text {core }}\right)$. Top, left to right: $I_{\mathrm{c}}, I_{\text {core }}$, polarization degree. Red contours trace high polarization degree, blue contours low $I_{\mathrm{c}}$. Middle: magnified view of the white rectangle marked above. Tick marks are in arcsec. Bottom: scatterplot of $I_{\mathrm{c}}$ and $I_{\text {core }}$. Red line: granulation simulation, blue dots: observation, black crosses: same after binning (see text for details).

\subsection{Simplified 1-D LTE synthesis of wave propagation}

In the next attempt to reproduce the observed correlation matrices, synthetic spectra were generated by assuming an upwards propagating wave that creates a temperature perturbation of the modified HSRA model as defined above. The perturbation was modeled as a Gaussian with a width of $\sigma=0.4$ in units of $\log$ $\tau$, and an amplitude $A$ that was scaled up with decreasing optical depth. We scaled the amplitude with the electron pressure that is related to the gas density using $A=A_{0} / \sqrt{p_{e l}}$ (see top panel of Fig. 9). We normalized the scaling law for the amplitude to 1 at $\log \tau=0$ to be able to prescribe exact initial values at this optical depth. We then synthesized spectra with the SIR code for the Gaussian perturbation moving through all 75 points of the optical depth grid $(\log \tau+1.4$ to -6 ; sampling 0.1 units of $\log \tau$ ), only modifying the temperature. The middle panel of Fig. 9 shows some of the temperature stratifications that result for an initial perturbation of $20 \mathrm{~K}$ at $\log \tau=0$. The propagation speed of the perturbation is constant in the optical depth scale, not in geometrical height, and without adding a corresponding velocity perturbation no Doppler shifts are induced.

Figure 10 compares a sample of observed profiles at a fixed spatial location over a time of around $600 \mathrm{~s}$ (left) with the synthetic spectra resulting when propagating an initial temperature
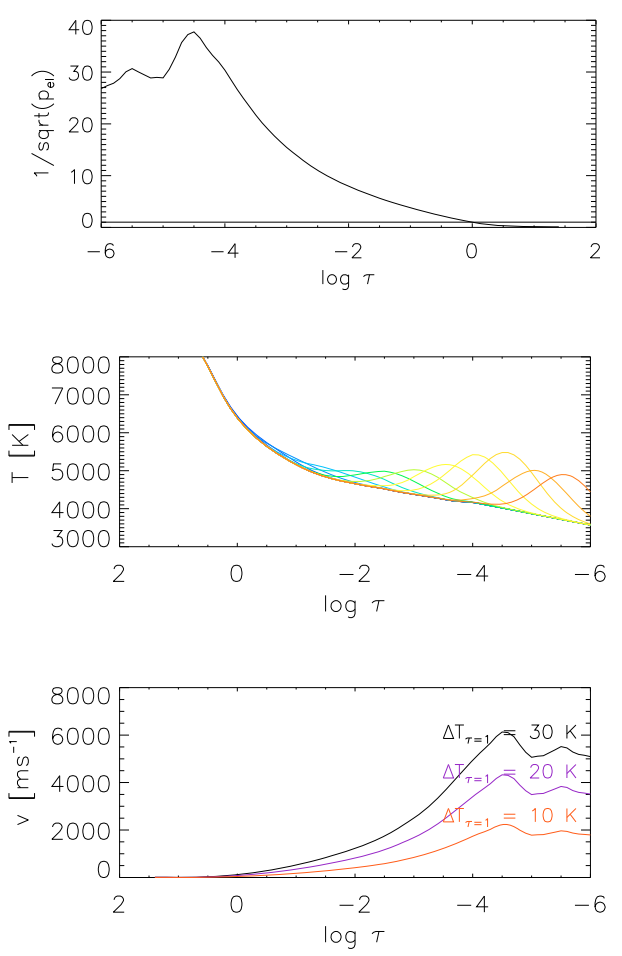

Fig. 9. Top: scaling law for the amplitudes of perturbations with optical depth. Middle: some of the temperature stratifications for the $20 \mathrm{~K}$ run. Bottom: velocity perturbation for three different temperature perturbations.

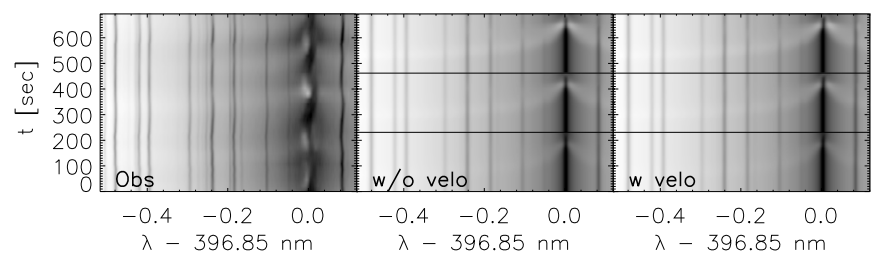

Fig. 10. Comparison of observed (left) and synthetic 1-D LTE spectra without velocities (middle), and with velocities included (right). The three runs with $10,20,30 \mathrm{~K}$ initial perturbation are shown contiguously from bottom to top in the simulated spectra.

perturbation of $10,20,30 \mathrm{~K}$ at $\log \tau=0$ through the optical depth grid (middle, from bottom to top for 10, 20, $30 \mathrm{~K}$ ). We synthesized the $\mathrm{Ca}$ II $\mathrm{H}$ line with all of its line blends for which atomic parameters were at hand. The photospheric lines at $630 \mathrm{~nm}$ were also synthesized to be conform with the standard wavelengths of POLIS.

The observed profiles have been stretched along the y-axis to match the evolution "speed" of the synthetic ones ${ }^{3}$. The brightenings originating in the line wing and culminating in strong emission in the core can be clearly traced in both the synthetic and observed spectra. The observed bright grains increase in intensity from the first to the second one like for the synthetic spectra, but this was actually only by chance; the observations were selected before the synthetic spectra were calculated. The complex behavior of the Ca line core in the observations is of course completely missing in the synthetic spectra due to the lack of velocities and the LTE assumption. The synthetic spectra are fully symmetric with respect to the Ca rest wavelength. Using the synthetic spectra for the three emulated "waves", we calculated the

\footnotetext{
3 The cadence of the observations was around $20 \mathrm{~s}$, yielding fewer spectra than in the wave simulation.
} 

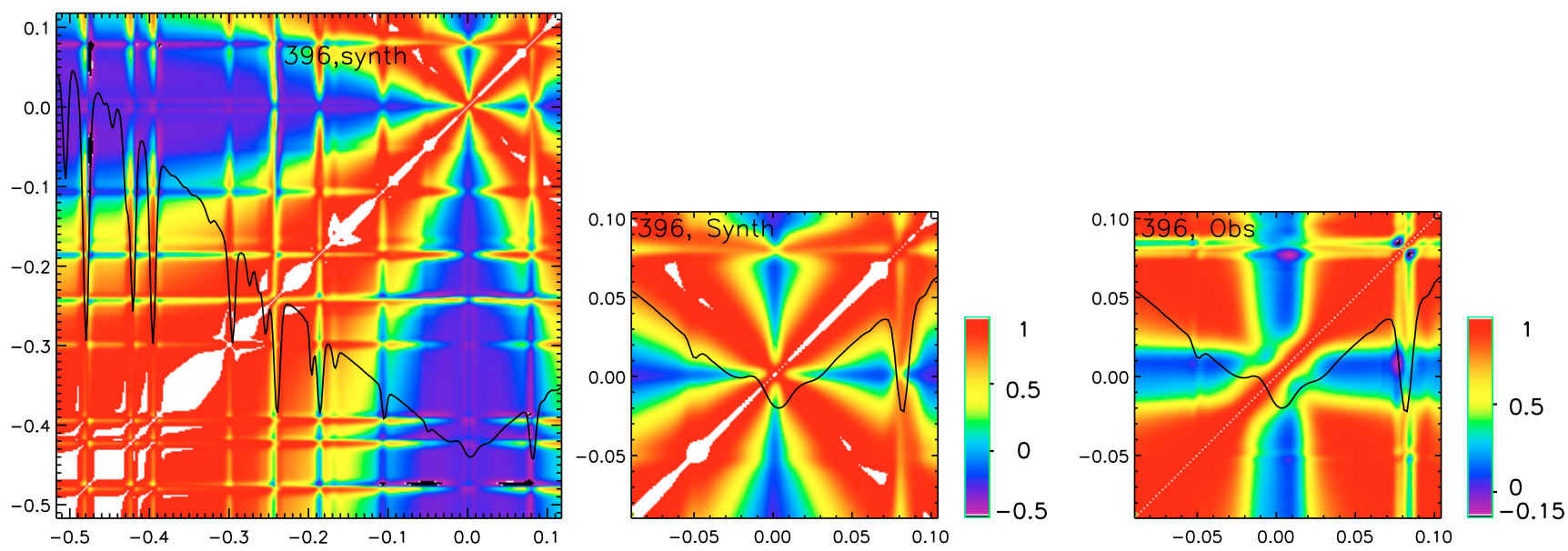

Fig. 11. Wavelength correlation matrices for Ca II H from the synthetic 1-D LTE spectra including a velocity perturbation. Left: full wavelength range. Middle: magnified view of the line core. Right: same section from observations. Wavelengths are $\lambda-396.85 \mathrm{~nm}$. The display range of the first two matrices is given by the left color bar, the one for the observations by the right color bar.

wavelength correlation matrices in the same way as for the observations. We remark that in this case only $3 \times 75$ spectra were used, as compared to the observations with several ten thousands of spectra, but the synthetic spectra cover all phases of the wave. This correlation matrix, however, turned out to be fully symmetric also for the photospheric lines, i.e., the pronounced red/blue asymmetry (cf. Fig. 2) was missing.

To improve the agreement with the observations, we then also introduced a line-of-sight velocity perturbation in phase with the temperature perturbation. The velocity amplitude was scaled with the same relation as the temperature perturbation (Fig. 9, lower panel). The velocity was chosen to be positive, i.e., a downflow, corresponding to a temperature increase by compression of gas during the propagation of an acoustic wave. This choice of direction for the velocity was necessary to produce the correct asymmetry for the photospheric lines, whereas for the $\mathrm{Ca}$ II $\mathrm{H}$ line core it enhances the red emission peak, contrary to what happens in observations (right panel of Fig. 10). If the velocity was applied with a time-lag, either leading or lagging behind the temperature increase, the resulting correlation matrix turned out to be fully symmetric again in the red and blue line wing.

Figures 11 and 12 show the correlation matrices for $\mathrm{Ca}$ II $\mathrm{H}$ and $630 \mathrm{~nm}$, respectively, obtained from the synthetic spectra including the positive velocity perturbation. They have to be compared with Figs. 2 and 3. Starting with the photospheric $630 \mathrm{~nm}$ lines, the shape of the asymmetry between red and blue wing can be reproduced by the assumed positive velocity. Similar to the observations, the correlation coefficient is lower when the intensity at the rest wavelength of the $630.15 \mathrm{~nm}$ line is correlated with other wavelengths than for the line core of the $630.25 \mathrm{~nm}$ line, which is presumably related to the line depth. The difference of the absolute values of the correlation coefficient between observations (minimum below -0.3) and the simulation (minimum $>0$ ) could be due to the granulation pattern in the observations that contributes anti-correlation for line-core wavelengths (Figs. 5 and 6), or instrumental effects, like for example the stray-light in the observations. If one forces the correlation at continuum wavelengths to be identical by reducing the correlation values obtained from the 1-D LTE spectra by 0.4 (red dashed line in bottom panel of Fig. 12), the wave simulation reproduces the magnitude of the observed asymmetry. For Ca II H, the global shape of the observed correlation matrix is roughly

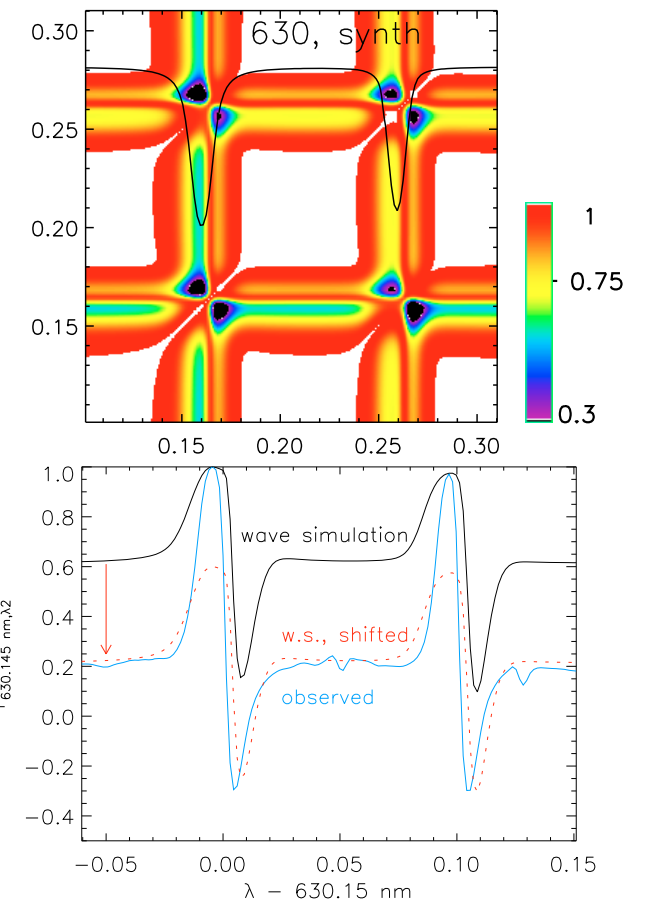

Fig. 12. Top: wavelength correlation matrix for $630 \mathrm{~nm}$ from the synthetic 1-D LTE spectra including a velocity perturbation. Wavelengths are $\lambda-630 \mathrm{~nm}$. Bottom: cuts through the correlation matrix at $630.145 \mathrm{~nm}$. Black: LTE wave simulation. Red dashed: same shifted by -0.4. Blue: observed.

reproduced, but with several huge differences to the observations. The band of high correlation around the diagonal widens monotonically with the wavelength distance to the $\mathrm{Ca}$ line core. Near the Ca line core, the half-circle/bridge between the stripes with the reduced correlation to the red and blue wing is missing; directly in the $\mathrm{Ca}$ line core, only one square of high correlation exists, located exactly at the rest wavelength of the Ca II $\mathrm{H}$ core.

The fine-structure near the very line core of $\mathrm{Ca}$ II $\mathrm{H}$ that was seen in the correlation matrix derived from the observations thus is not reproduced by the simplified wave simulation. A close match is however not to be expected in this case, since the processes in the upper atmosphere during the passage of a shock front are very complex. They include large Doppler shifts of 


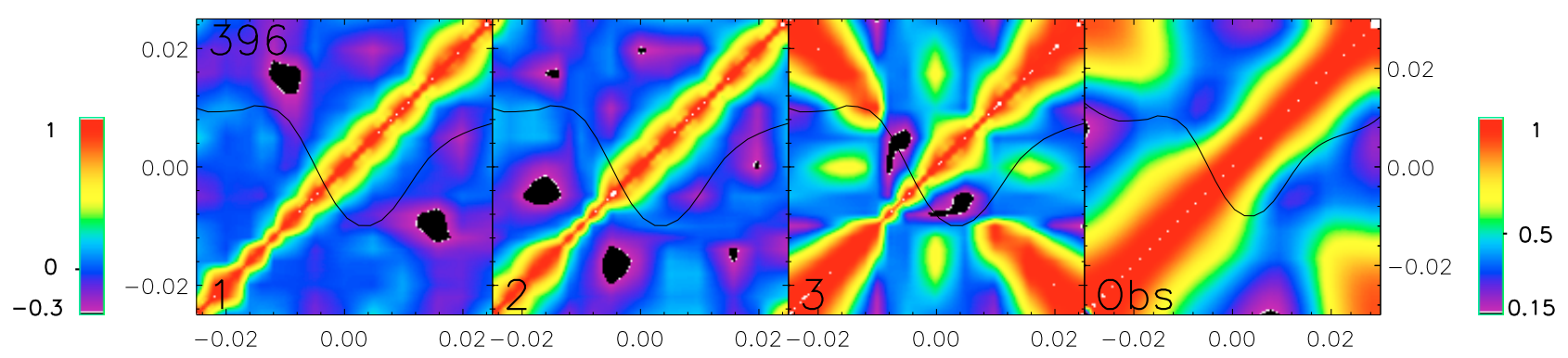

Fig. 13. Left to right: correlation matrices of the Ca II $\mathrm{H}$ line core from 1-D NLTE calculations for stochastic waves $(1,2)$, monochromatic waves (3), corresponding section of matrix from observations. Wavelength range $(-0.03 \mathrm{~nm}$ to $0.03 \mathrm{~nm})$ and display range (0.15 to 1$)$ of the observations' matrix are slightly different. The right color bar shows the display range for the observations (4th column), the left for the other three matrices. Wavelengths are $\lambda-396.849 \mathrm{~nm}$.

the line due to upwards and downwards directed motions, together with strong variations of the intensity near the line core, where the flow directions in front of and behind the shock front can be going in opposite directions (Carlsson \& Stein 1997). Macroscopic mass flows in the atmosphere correspond to the correlation with a shifted line profile that yields the same correlation matrix, but displaced in wavelength, if the profile shape is not changed. To test the effect of macroscopic flows in addition to the simulated wave, we took the correlation matrix for the synthetic Ca II H spectra of the wave simulation, shifted it along the diagonal, and added it to the original un-shifted one. Shifting the correlation matrix by around $5 \mathrm{kms}^{-1}$ and adding it to the original matrix reproduced the features of the observed correlation matrix near the line core, i.e., the double squares of high correlation and connecting bridge between low correlation stripes, but it also doubled the pattern due to the photospheric blends in full contradiction to the observations. We thus suggest that any additional macroscopic flows should only be present in the upper atmospheric layers not seen by the photospheric blends, and that the fine-structure in the correlation matrix near the $\mathrm{Ca}$ II $\mathrm{H}$ core could be produced by the complex flow pattern near a shock front that passes through the atmosphere.

The two contributions from the granulation simulation and the simplified wave propagation together seem to be able to reproduce the patterns seen in the correlation matrix of observed photospheric spectra and the global structure of the Ca II H correlation matrix, albeit not the fine structure near the line core of the chromospheric line.

\subsection{NLTE 1-D simulations}

As a third numerical experiment, we used spectra generated from 1-D NLTE simulations done with the WAVE code (Rammacher \& Ulmschneider 2003). The simulations were performed similar to Carlsson \& Stein (1997) with a photospheric piston that excites acoustic waves of various types. The driver of the piston was varied between stochastic excitation and monochromatic waves. Here we used three runs corresponding to stochastic waves with a mechanical energy flux of $5 \times 10^{7} \mathrm{erg} \mathrm{cm}^{-2} \mathrm{~s}^{-1}$ and $2 \times 10^{8} \mathrm{erg} \mathrm{cm}^{-2} \mathrm{~s}^{-1}$, and monochromatic waves of $45 \mathrm{~s}$ period with also $2 \times 10^{8} \mathrm{erg} \mathrm{cm}^{-2} \mathrm{~s}^{-1}$ as energy flux.

The correct calculation of spectra for the chromospheric emission lines requires the use of partial redistribution (PRD). However, a line treatment implementing PRD like in Ulmschneider (1994) leads to excessive computation times that cannot be tolerated in time-dependent wave calculations. We thus followed Huenerth \& Ulmschneider (1995) by employing a so-called "pseudo-PRD", which computes the line assuming complete frequency redistribution (CRD) but then artificially removes the damping wings from the lines by multiplying the damping parameter in the Voigt function by a factor of $1 / 100$. The spectra were calculated with this method from the full simulation runs only for the very core of $\mathrm{Ca}$ II $\mathrm{H}$ in a range of $\pm 0.03 \mathrm{~nm}$ around the rest wavelength, but this covers the finestructure as seen in the observations. Spectral samples of 1500 such profiles were used to calculate the correlation matrices for each piston model.

Figure 13 shows the resulting correlation matrices for the runs with the stochastic excitation (1 and 2) and the monochromatic case (3). The patterns in the correlation matrices for the stochastic excitation do not match the observations at all (last column in Fig. 13). The matrix for the monochromatic waves, however, partly fits that of the observed spectra. The central band of high correlation is smaller than for the observations, and there exists a cross-like structure of higher correlation values at the rest wavelength, but the high correlation values at $\pm 0.025 \mathrm{~nm}$ appear in both observations and simulations. The bridge/halfcircle that connects the two bands with reduced correlation starting from the core is present in the simulations as well. Some of the additional fine-structure in the simulations' correlation matrix is missing in the observations, but this could actually also be due to either limitations on the observational side (tempo$\mathrm{ral} / \mathrm{spatial} / \mathrm{spectral}$ resolution) or to the realism of the simulations with a strictly monochromatic permanent driver.

To investigate the correlation matrix for the 1-D NLTE simulations in the line wings as well, new spectra were synthesized from the simulation results using the PRD code as described in Ulmschneider (1994). This allowed us to extend the wavelength range, but unfortunately is quite demanding on computing power. So far, only a set of 100 spectra with three seconds of temporal sampling are available for the case of the monochromatic 45-s piston (Fig. 14, top). The spectra were calculated on a non-equidistant wavelength grid with a spectral sampling of around $4 \mathrm{pm}$ in the line wing and $1.5 \mathrm{pm}$ near the line core to reduce the computational effort. We interpolated and resampled the spectra to an equidistant sampling of $2 \mathrm{pm}$ for a better match to the POLIS observations ( $1.9 \mathrm{pm}$ sampling at a spectral resolution of 220.000, Beck et al. 2005) and then determined the correlation matrix as before. The bottom left and right panel in Fig. 14 show the resulting correlation matrix for the full wavelength range and a magnification of the line core, respectively. Similar to the simplified 1-D LTE modeling, the correlation band around the diagonal widens monotonically for an increasing distance from the line core, contrary to the observations (cf. Fig. 3). The correlation from the core to the red and blue wing $\left(r_{0, \lambda_{i}}\right.$ and $r_{\lambda_{i}, 0}$ ) now shows a positive correlation for $\left|\lambda_{i}\right|>0.1 \mathrm{~nm}$, contrary 

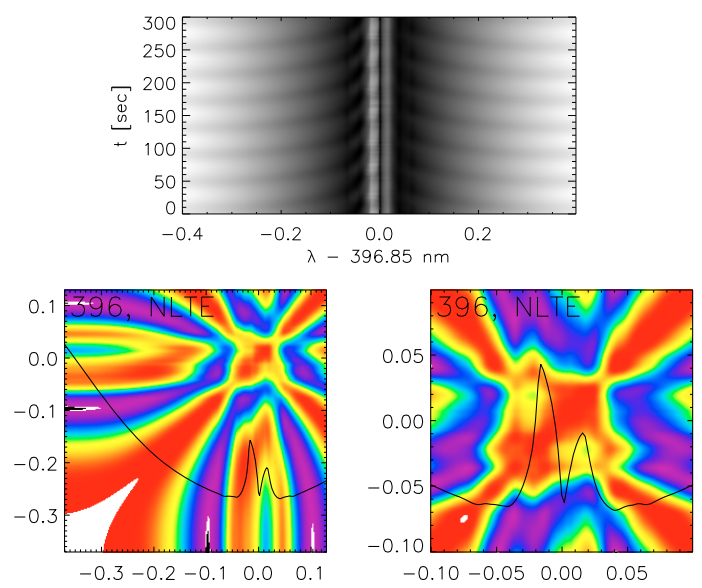

Fig. 14. Top: 1-D NLTE spectra for monochromatic waves. Bottom left: correlation matrix of Ca II H. Bottom right: magnification of line core. Wavelengths are $\lambda-396.849 \mathrm{~nm}$. Display range of the correlation matrices is \pm 1 .

to both the observations and the simplified LTE modeling. This could be due to the short time span sampled and the strict period of the driver; the correlation of a spectrally un-smoothed version of the spectra without resampling shows several repeated stripes of alternating high and low correlation there. The line core region (right) matches the observations better (cp. to the rightmost panel in Fig. 11). The two squares of high correlation at $\pm 0.02 \mathrm{~nm}$ on the diagonal are present as well as the bridge between the stripes with the reduced correlation to the red and blue wing, albeit the correlation only drops to zero and does not turn to anti-correlation $\left(r_{ \pm 0.02, \mp 0.02}\right)$.

In total, the 1-D LTE or NLTE calculations are able to reproduce the general shape of the correlation matrix of $\mathrm{Ca}$ II $\mathrm{H}$ in the line wing, whereas the 1-D NLTE calculation yields a first order match to the very line core. In both cases, the lower part of the atmosphere is treated similarly as being permeated by propagating acoustic waves leading to similar spectral patterns (cp. the spectra in Figs. 10 and 14). The line core region is only reproduced in the NLTE calculations due to the complex dynamics in the shock fronts that form in the upper atmosphere (e.g., Rammacher \& Ulmschneider 1992; Carlsson \& Stein 1997). The remaining mismatch between observed and synthetic correlation matrices is presumably due to the granulation pattern which is absent in the synthetic spectra of the wave simulations.

\subsection{3-D MHD simulation}

As a final numerical model, we obtained spectra in the $1565 \mathrm{~nm}$ and $630 \mathrm{~nm}$ range from a 3-D MHD simulation run done with the $\mathrm{CO}^{5} \mathrm{BOLD}$ code (Freytag et al. 2002). The simulations are described in more detail in Schaffenberger et al. $(2005,2006)$; the simulation box had an extension of $4.8 \mathrm{Mm} \times 4.8 \mathrm{Mm}$ in the horizontal domain. The spectra were calculated for a single snapshot of a simulation run that included magnetic fields; the dynamics in the upper atmosphere is, however, dominated by dynamic events and shock fronts like in the field-free case (cf. Wedemeyer et al. 2004). The radiative transfer is treated by mean opacities in the simulation, which prevents to obtain NLTE spectra of for instance the chromospheric Ca II lines directly from the simulation results without additional calculations; we thus only used the photospheric spectra of Fe I $630.15 \mathrm{~nm}, 630.25,1564.8 \mathrm{~nm}$ and $1565.2 \mathrm{~nm}$ which can be synthesized at once. Figure 15 shows the correlation matrices for these four spectral lines obtained
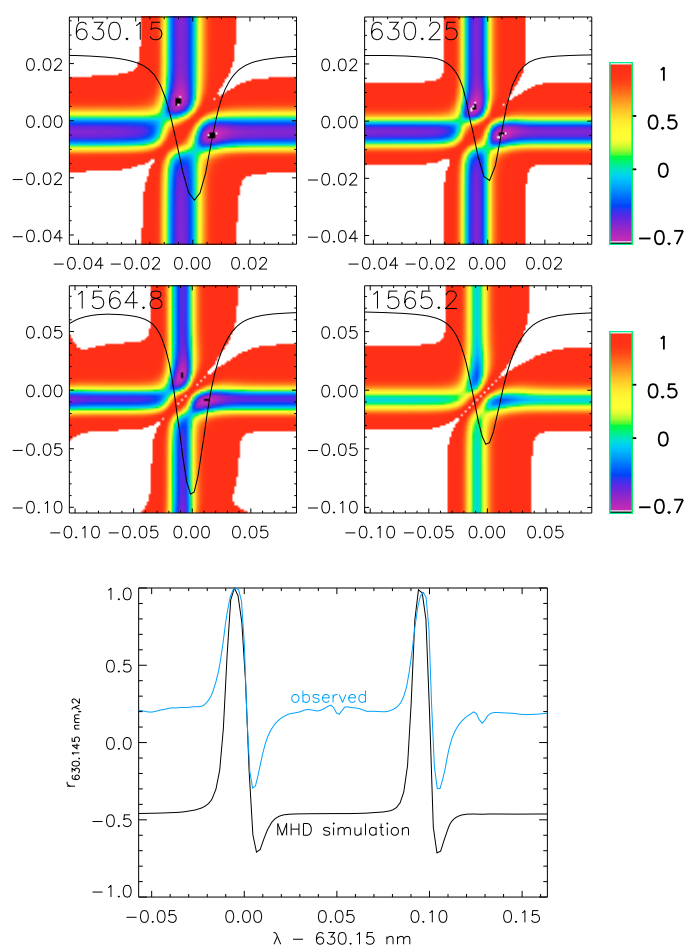

Fig. 15. Top four panels: correlation matrices from a 3-D MHD simulation for $630.15 \mathrm{~nm}$ (top left), $630.25 \mathrm{~nm}$ (top right), $1564.8 \mathrm{~nm}$ (bottom left), and $1565.2 \mathrm{~nm}$ (bottom right). Wavelengths in $\mathrm{nm}$ are relative to the rest wavelength of the respective line. Bottom panel: cuts through the correlation matrix at $630.145 \mathrm{~nm}$. Black: MHD simulation. Blue: observed.

from the 3-D MHD simulation (cp. to Fig. 2). The structure of the correlation matrix from the observed spectra is reproduced accurately: the lines with a larger line depth $(630.15 / 1564.8 \mathrm{~nm})$ show a stronger reduction of the correlation, their stripes with the reduced correlation are broader, and all lines in the matrix from the simulation show the correct red/blue asymmetry. The sole difference to the observations is the magnitude of the correlation coefficients: the simulation generally shows stronger anticorrelation (display range from -0.7 instead of -0.3 like for the observations). The global shape of the correlation matrix from the 3-D MHD simulation matches the observations much better than the simplified 1-D LTE modeling of the wave propagation, again presumably due to the absence of the granulation pattern in the latter. The reduction of the correlation to the red of the line core is weaker in the MHD simulation than in the observations, and at continuum wavelengths the correlation is much lower (bottom panel of Fig. 15). The MHD simulation is thus in some sense between the "pure" granulation simulation (largest anti-correlation at continuum wavelengths without asymmetry) and the 1-D LTE wave simulation (positive correlation at continuum wavelengths and largest asymmetry). This could be due to the fact that the 3-D MHD simulations include granulation, but miss one strong source of oscillations and waves: the large-scale solar oscillations of the p-modes, which are not present in the simulation box.

\section{Correlation matrices for temperature}

To highlight the possible usefulness of the correlation matrix for an analysis also of other physical quantities than spectra, we calculated correlation matrices for temperature as a function of 

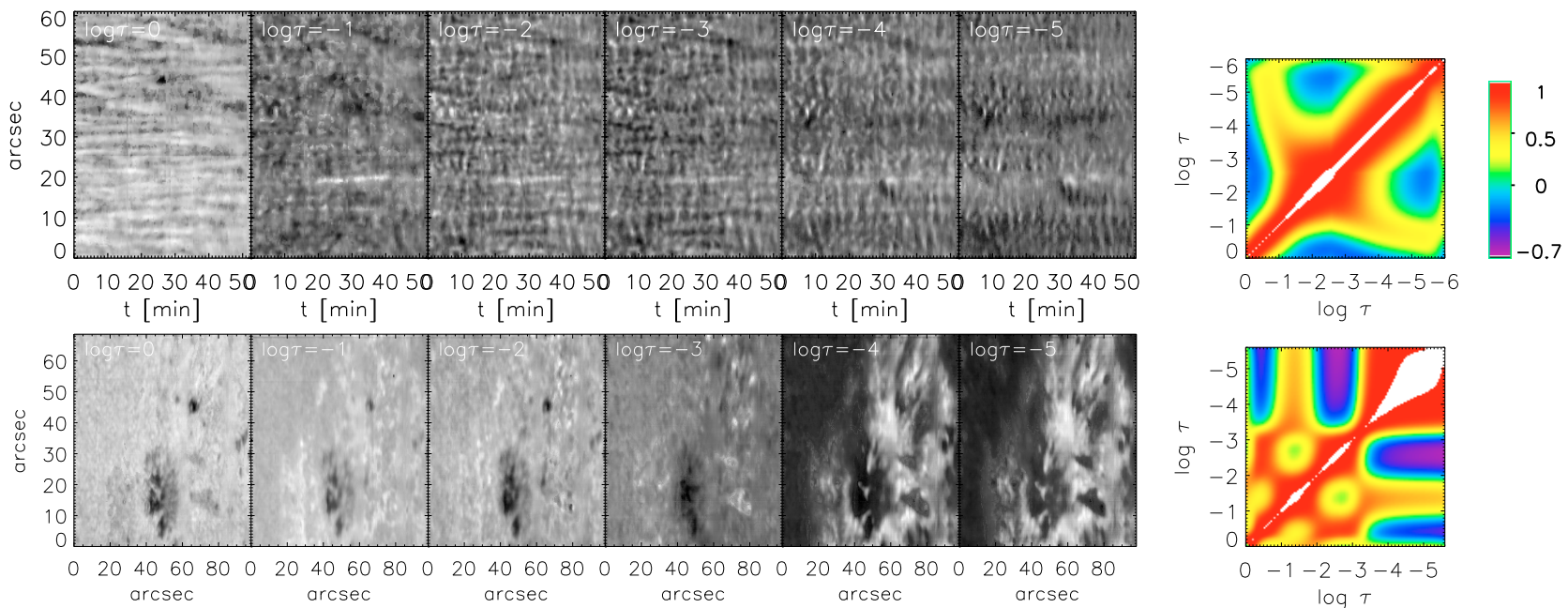

$\log \tau$
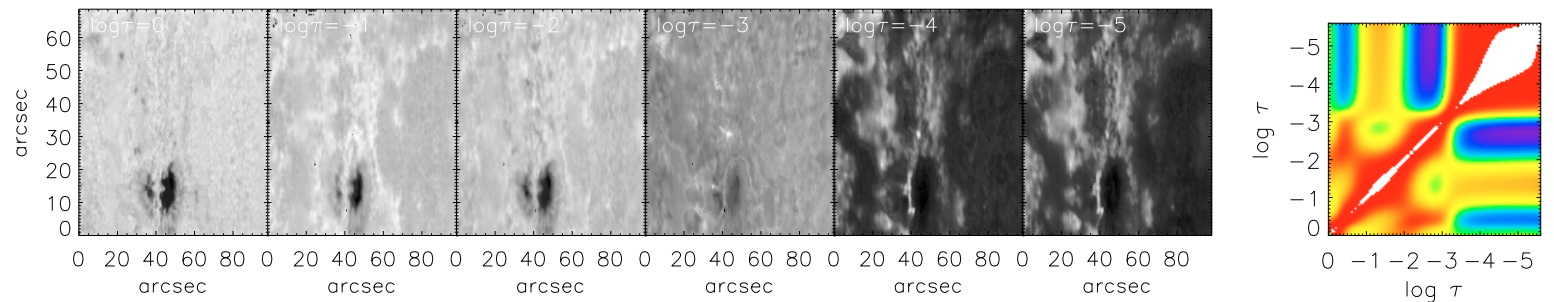

$\log \tau$
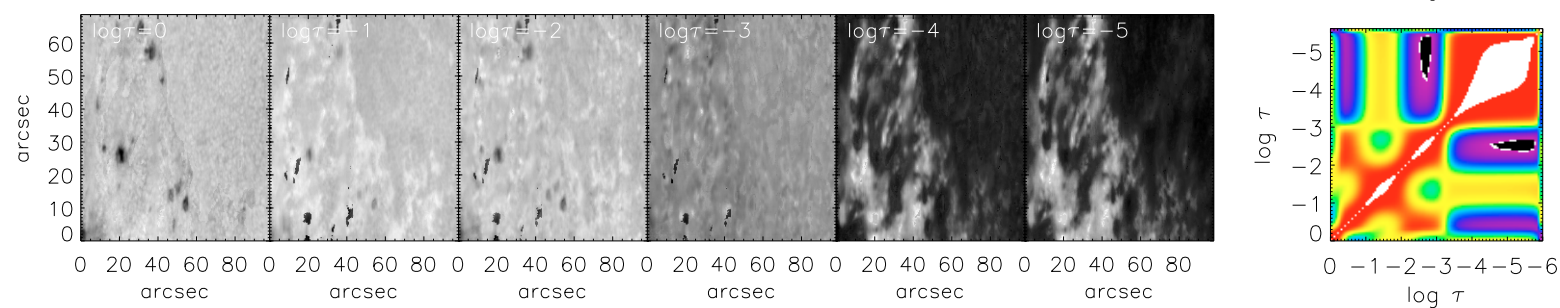

Fig. 16. Left top: temperature maps at various optical depths from a LTE inversion of Ca II H spectra of a time-series. Right top: correlation matrix of temperature as a function of optical depth. Bottom three rows: same for three scans of an active region off the disk center. All correlation matrices use the display range shown at right top.

optical depth. The temperature values were derived by an inversion of Ca II H spectra of a 1-h time-series (cf. Beck et al. 2008, observation no. 21 in the present paper, cf. Appendix A.1) and three scans of an active region off the disk center (taken on $08 / 12 / 2007, \theta \sim 50^{\circ}$; not listed in the appendix). The inversion is based on the SIR code in synthesis mode (Ruiz Cobo \& del Toro Iniesta 1992; Ruiz Cobo 1998); it will be discussed in more detail in a forthcoming paper. It mainly differs from the standard version of SIR in the usage of a pre-calculated fixed intensitytemperature response function and the initial use of an archive of pre-calculated $\mathrm{Ca}$ spectra. The use of the archive was found to be necessary to achieve a reasonable match of the $\mathrm{Ca}$ line core region; the standard iterative approach of SIR failed there because of the complex temperature stratifications required. The inversion with the LTE assumption basically maps the intensity values at a given wavelength in the $\mathrm{Ca}$ profile to some range in optical depth, as given by the intensity contribution function (cf. Fig. 5 of Rezaei et al. 2008; Beck et al. 2009).

Figure 16 shows the resulting temperature maps at six levels $(\log \tau=0,-1, \ldots,-5)$ at left. The temperature maps of the timeseries are to first order identical to the intensities in the spectral windows $\mathrm{OW}, \mathrm{MW} 2, \mathrm{MW} 3$, IW1 $\mathrm{H}_{2} \mathrm{~V}$ and $\mathrm{Ca}$ line core in Beck et al. (2008, Fig. 4). Correlating the maps of $T\left(\tau_{i}, x, t\right)$ and $T\left(\tau_{j}, x, t\right)$ using Eq. (1) yielded the correlation matrices shown at the right in Fig. 16. For the time-series, the shape of the temperature correlation does not match well to any pattern in the Ca II H matrix, but the matrix of Ca II IR at $854 \mathrm{~nm}$ (bottom row in Fig. 4) shows some similarity. The structure seen from $\Delta \lambda=-0.05 \mathrm{~nm}$ to $0 \mathrm{~nm}$ in the matrix from the spectra matches that from $\log \tau=-3$ to -6 in the temperature: an iterative sequence of increased and reduced width of the high correlation band around the diagonal with lower correlation at, e.g., $r_{\Delta \lambda=-0.05,0}$ and $r_{\log \tau=-3,-5}$, respectively. The matrices from the temperature maps of the active region are similar to each other, but differ from the one for the quiet Sun, which will be due to the different heliocentric angle and the solar surface structure, like for the two observations of Ca II IR $854 \mathrm{~nm}$ that yielded quite different correlation matrices. They only keep a few common properties, like for instance the anti-correlation at around $\log \tau=-2.5$ to all regions above. A conversion from $\log \tau$ to the corresponding wavelength (or vice versa) will be needed for a direct comparison with the correlation matrices from spectra, or a conversion from $T(\tau)$ to geometrical height for a comparison with simulations, but the inversion code has first to be improved for a quantitative analysis.

\section{Summary and discussion}

We have calculated the linear correlation coefficient between intensities at different wavelengths for various photospheric and 
chromospheric spectral lines from near-UV to near-IR wavelengths. The correlation coefficients yield a matrix that contains information on the causal relationship between different wavelengths and thus height layers in the solar atmosphere. For all photospheric spectral lines, a pronounced asymmetry between the red and blue wing is found. The intensity at the line core has a weaker correlation with the intensities in the red wing than with those in the blue one. Correlations between the intensities at continuum wavelengths are always high. All chromospheric spectral lines considered (Ca II H,Ca II IR at $854 \mathrm{~nm}$ and $866 \mathrm{~nm}$ ) show much more structure in the correlation matrix near their respective line cores than the photospheric lines.

A simple granulation simulation that assumes hot upflows and cold downflows produces a strong reduction of the correlation between the line-core intensities of photospheric lines and the intensity at continuum wavelengths. The resulting correlation matrix, however, is nearly fully symmetric in the red and blue wing when the velocity is directly proportional to the intensity. Only when a deviation from the linear relationship is introduced, e.g. by adding a random velocity, the correlation matrix shows an asymmetry between the red and blue wing. The actually observed velocities show a strong scatter around a linear relationship between the continuum intensity and velocity. Synthetic LTE spectra corresponding to the upwards passage of a temperature perturbation are able to reproduce the additional signature seen in the photospheric lines, the pronounced asymmetry between the red and blue wing, if a positive velocity (downflow) is introduced together with the temperature enhancement. Spectra of Ca II H synthesized from 1-D NLTE simulations, where propagating waves are generated by a photospheric piston, yield a correlation matrix that qualitatively matches the pattern in the correlation matrix of observed spectra near the very line core of $\mathrm{Ca}$ II $\mathrm{H}$, but only for the case of a monochromatic driver with a $45 \mathrm{~s}$ period. Stochastic excitation of the piston produces a correlation matrix that is inconsistent with the observations. A 3-D MHD simulation run, in which the dynamics in the upper atmosphere is dominated by shock waves, faithfully reproduces the asymmetry of the red and blue wing seen for all photospheric lines, together with the relative amount of correlation that seems to depend on the line depth of the respective lines. The 3-D simulation contains contributions from both granulation and waves. Temperature maps at different optical depth layers that were derived from an LTE inversion of observed spectra yield a correlation matrix that has at least a structural resemblance to the matrices obtained from observed spectra of Ca II IR $854 \mathrm{~nm}$. A clear difference between the quiet Sun and active regions is seen in the temperature correlation and the correlation matrices obtained from observations.

Influence of granulation pattern: The granulation pattern leaves its imprint on the observed correlation matrices in all photospheric spectral lines, which partially hides the signature of the dynamic processes relevant for the chromosphere. The observed asymmetry between the intensities of the red and blue wing, however, could not be related to the granulation pattern (Sect. 4.1). To better isolate the dynamic processes, it seems advisable to partly remove the contribution of the granulation pattern from the spectra prior to the calculation of wavelength correlation matrices in future studies. For large-area maps, this could be achieved by a local (or global) adjustment of continuum intensity values. One could force all spectra in a small-scale $\left(1-3^{\prime \prime}\right.$ radius) surrounding of each pixel to an identical intensity in continuum wavelengths, corresponding to removing the granulation pattern locally, and then calculate several "local" correlation matrices to be averaged later on, or force the continuum intensity to unity across the full FOV. Time-series will be even better suited for the removal of the granulation pattern, because the granulation can be more accurately filtered in the temporal than in the spatial domain: the typical time-scale of granules $(5 \mathrm{~min}$ ) differs from the fast evolving and propagating waves (around 1 min typical life time from first visibility in continuum layers to shock signature in the chromosphere, e.g., Beck et al. 2008), whereas the spatial scales of granules and chromospheric brightenings can be of a comparable size. The signature of the dynamic processes will then become more prominent in granulation-filtered data. Preliminary tests with e.g. the method of local intensity balancing significantly reduced the areas of high correlation around the diagonal.

\section{Conclusions}

The matrices of the linear correlation coefficient of photospheric and chromospheric spectral lines carry information on the physical processes in the solar atmosphere. They are influenced by both the convective granulation pattern and more transient events like propagating waves that are presumably related to the chromospheric heating process. We have shown that the most pronounced feature in the correlation matrices of photospheric lines, an asymmetry between the intensities in the red and blue wing, can be reproduced to first order by assuming propagating (acoustic) waves that produce an intensity enhancement together with a red-shift of the spectral lines. Such propagating waves also qualitatively reproduce the correlation matrix of chromospheric spectral lines.

The calculation of the correlation matrices can be applied to other physical quantities as well, like for example temperature maps at different height levels in the solar atmosphere. The method seems suited to compare observations of chromospheric spectral lines, where NLTE conditions apply, with theoretical or numerical modeling of wave propagation in the solar atmosphere. The analysis of observed spectra can be refined by filtering out the signature of the granulation pattern that differs mainly in its temporal behavior from the more transient chromospheric heating process. Other physical quantities like 3-D temperature cubes can be analyzed with the correlation method as well, which seems to be a useful tool for a comparison of simulations and observations.

Acknowledgements. The authors want to thank F. Wöger (NSO) and A. Tritschler (NSO) for the IBIS data set at $854 \mathrm{~nm}$. Similar thanks go to O. Steiner (KIS) and R. Rezaei (KIS) for the 3-D MHD spectra. The VTT is operated by the Kiepenheuer-Institut für Sonnenphysik (KIS) at the Spanish Observatorio del Teide of the Instituto de Astrofísica de Canarias (IAC). The POLIS instrument has been a joint development of the High Altitude Observatory (Boulder, USA) and the KIS. The National Solar Observatory (NSO) is operated by the Association of Universities for Research in Astronomy, Inc. (AURA), under cooperative agreement with the National Science Foundation. IBIS has been built by INAF/Osservatorio Astrofisico di Arcetri with contributions from the Universities of Firenze and Roma "Tor Vergata", the NSO, and the Italian Ministries of Research (MIUR) and Foreign Affairs (MAE). W.R. acknowledges support by the Deutsche Forschungsgemeinschaft under grant SCHM 1168/6-1. C.B. acknowledges partial support by the Spanish Ministry of Science and Innovation through project AYA 2007-63881.

C.B. wants to apologize sincerely for not having cited Liu's important work before, he was not aware of its existence. 

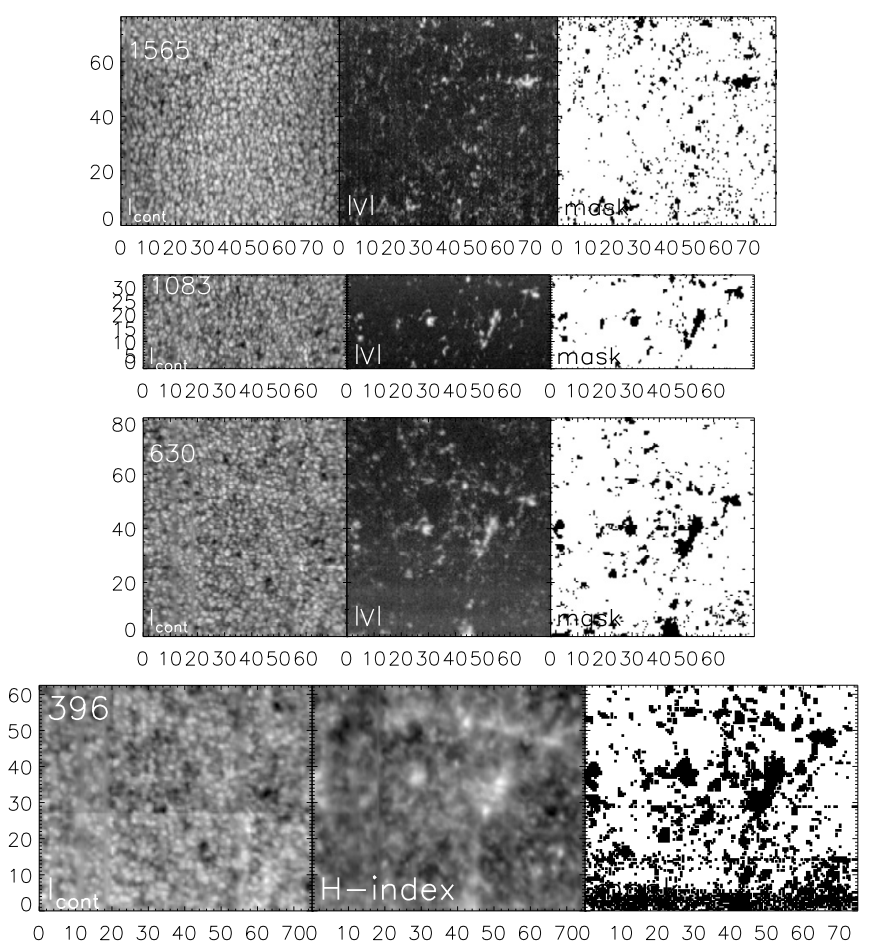

Fig. A.1. Examples of the large-area scans used. Left to right: continuum intensity, absolute integrated Stokes $V$ signal, mask of magnetic fields. Top to bottom: 1565-6 (see. Table A.1 for the details, the number -X denotes the observation); 1083-7; 630-10; 396-15. For Ca II H, the integrated line core emission (H-index) is shown instead of $V$. Tick marks are in arcsec.

\section{Appendix A: Observations used}

\section{A.1. TIP/POLIS}

For the derivation of the correlation matrices, several data sets taken with the spectropolarimeters TIP and POLIS were used. Table A.1 lists the data sets with a consecutive number, the operation number of the data set on the day, the date, the total integration time per slit position, and the approximate extent of the scanned area. For the polarimetric data sets (TIP at $1083 \mathrm{~nm}$ or $1565 \mathrm{~nm}$, POLIS at $630 \mathrm{~nm}$ ), we show a continuum intensity map at the left, the absolute wavelength integrated circular polarization in the middle, and the mask of field-free and magnetic locations derived from the polarization signal at right. For the TIP data in the $1083 \mathrm{~nm}$ range, the polarization signal of the photospheric Si I line at $1082.7 \mathrm{~nm}$ was used to define the masks. For the Ca II $\mathrm{H}$ data from POLIS, the intensity integrated over $0.1 \mathrm{~nm}$ around the Ca line core (H-index) is shown in the middle. The mask in that case has been created from the simultaneous $630 \mathrm{~nm}$ data of the second POLIS channel. The maps for the time-series used here (Fig. A.2) are organized in the same way as for the large-area scans. Spatial sampling in scanning direction was 0.5 , along the slit the sampling was $0 . ' 15(630 \mathrm{~nm}), 0 . ' 29$ $(396 \mathrm{~nm}), 0.17$ (1083 and $1565 \mathrm{~nm})$. Additional information on the respective data sets can be found in their online overview archives (accessible from http://wWw.kis.uni-freiburg. de $\longrightarrow$ Observatories $\longrightarrow$ Data archives).

\section{A.2. Ca II IR}

The spectroscopic data of Ca II IR $866 \mathrm{~nm}$ was taken at the VTT using a PCO camera at the main spectrograph. Simultaneously

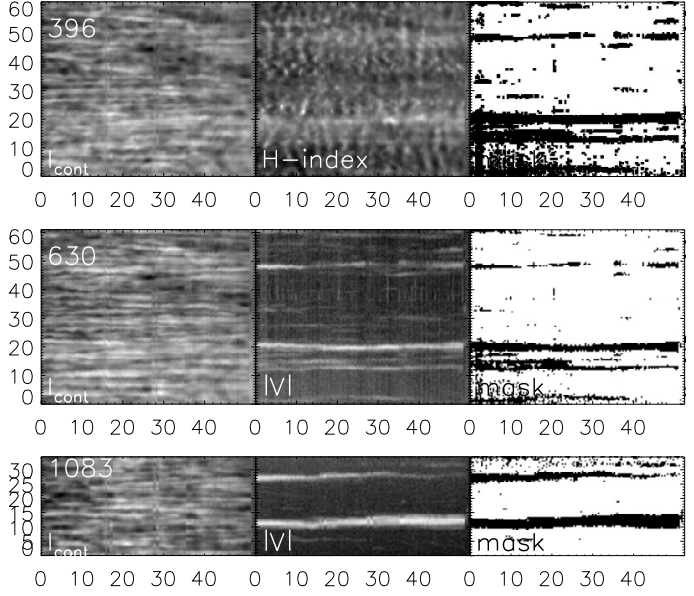

Fig. A.2. Overview of the time-series used, same layout as Fig. A.1. Top to bottom: 396-21, 630-22, 1083-23. The $y$-axis is in arcsec, the $x$-axis gives the time in minutes.

Table A.1. Detailed list of the observations used. The operation number ( 2 nd column) is the number of the observation on that day.

\begin{tabular}{llllll}
\hline \hline No. & $\begin{array}{l}\text { Op. } \\
\text { no. }\end{array}$ & $\begin{array}{l}\text { Wavelength } \\
\text { nm }\end{array}$ & ddmmyy & $\begin{array}{l}\text { Integ. } \\
\text { time }\end{array}$ & $\begin{array}{l}\text { Size x/y } \\
\text { in arcsec }\end{array}$ \\
\hline 1 & 3 & 1565 & $10 / 08 / 06$ & $30 \mathrm{~s}$ & $35 / 70$ \\
2 & 3 & 1565 & $12 / 08 / 06$ & 10 & $120 / 70$ \\
3 & 1 & 1565 & $14 / 08 / 06$ & 10 & $200 / 70$ \\
4 & 1 & 1565 & $21 / 08 / 06$ & 6 & $75 / 70$ \\
5 & 1 & 1565 & $07 / 09 / 07$ & 5 & $80 / 70$ \\
6 & 7 & 1565 & $07 / 09 / 07$ & 5 & $80 / 70$ \\
7 & 5 & 1083 & $24 / 07 / 06$ & 6.5 & $75 / 35$ \\
8 & 6 & 1083 & $24 / 07 / 06$ & 6.5 & $75 / 35$ \\
9 & 7 & 1083 & $24 / 07 / 06$ & 6.5 & $75 / 35$ \\
10 & 6 & 630 & $24 / 07 / 06$ & 6.5 & $75 / 87$ \\
11 & 7 & 630 & $24 / 07 / 06$ & 6.5 & $75 / 87$ \\
12 & 8 & 630 & $24 / 07 / 06$ & 6.5 & $75 / 87$ \\
13 & 2 & 396 & $18 / 07 / 06$ & 6.5 & $40 / 63$ \\
14 & 3 & 396 & $18 / 07 / 06$ & 6.5 & $40 / 63$ \\
15 & 6 & 396 & $24 / 07 / 06$ & 6.5 & $75 / 63$ \\
16 & 7 & 396 & $24 / 07 / 06$ & 6.5 & $75 / 63$ \\
17 & 9 & 396 & $08 / 12 / 07$ & 3.3 & $100 / 63$ \\
18 & - & 866 & $20 / 06 / 07$ & 0.25 & $120 / 177$ \\
19 & 0 & 854 & $23 / 09 / 08$ & $100 \mathrm{~m} \mathrm{~s}{ }^{1}$ & $39 / 72$ \\
20 & - & 854 & $29 / 08 / 09$ & $25 \mathrm{~s}$ & $60 / 84$ \\
21 & 1 & 396 & $24 / 07 / 06$ & 3.3 & $52^{2} / 63$ \\
22 & 1 & 630 & $24 / 07 / 06$ & 3.3 & $52^{2} / 63$ \\
23 & 1 & 1083 & $24 / 07 / 06$ & 3 & $52^{2} / 35$ \\
\hline
\end{tabular}

Notes. ${ }^{(1)}$ IBIS data, exposure time per wavelength; ${ }^{(2)}$ duration in min.

with the Ca II IR $866 \mathrm{~nm}$ line also Ca II H was observed. A study of center-to-limb variation and some time series are available from the observation campaign and can be used in further investigations. As no polarimetric data is available in this case, Fig. A.3 only shows a pseudo-continuum map of the intensity in the line wing and the wavelength integrated line core intensity comparable to the $\mathrm{H}$-index for $\mathrm{Ca}$ II $\mathrm{H}$.

For Ca II IR $854 \mathrm{~nm}$, we used two different data sets. The first map (Ca II IR 854-20) was obtained again at the VTT using a PCO camera at the main spectrograph. The camera was run in parallel to TIP in He I $1083 \mathrm{~nm}$ and POLIS in its standard configuration; the data were taken in the quiet Sun at disk center. We have not yet aligned the data of all the instruments, so we have not been able to create a mask of locations with significant 

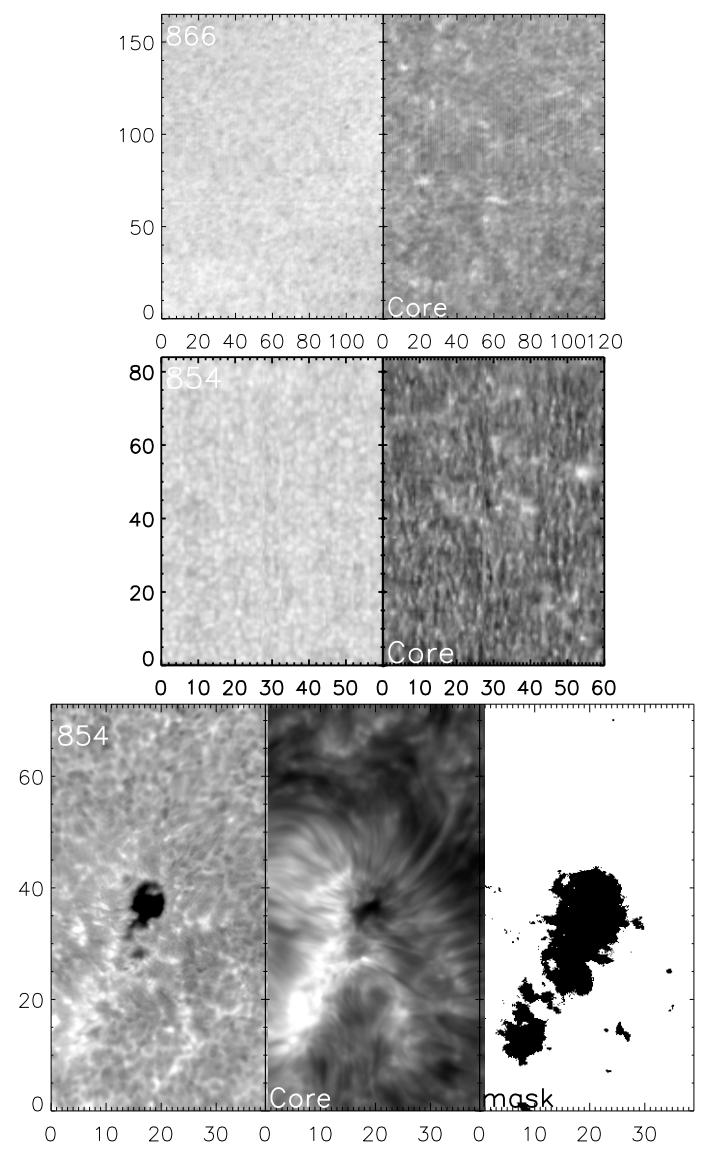

Fig. A.3. Top: overview of the Ca II IR map 866-18. Left: wing intensity, right: core intensity. Middle: overview of the Ca II IR map 854-20. Left: wing intensity, right: core intensity. Bottom: overview of the CaII IR map 854-19. Left to right: wing intensity, core intensity, mask. Tick marks are in arcsec.

polarization signal and used the full FOV instead. The second data set for Ca II IR $854 \mathrm{~nm}$ was taken in September 2008 with the IBIS spectrometer at the Dunn Solar Telescope in Sac Peak/NM. The instrument was used in the spectropolarimetric mode. The mask was in this case derived from the absolute integrated Stokes $V$ signal of Ca II IR $854 \mathrm{~nm}$ for simplicity; a second data set in $630.25 \mathrm{~nm}$ taken two minutes later is also available. The $854 \mathrm{~nm}$ line was scanned in 30 steps with a spectral sampling of $4.3 \mathrm{pm}$; spatial sampling was 0 !' 17 . The data set was on an active region containing a pore; it is not fully compatible to the quiet Sun observations on disk center that were used for all other spectral lines.

\section{References}

Beck, C., \& Rezaei, R. 2009, A\&A, 502, 969

Beck, C., Schmidt, W., Kentischer, T., et al. 2005, A\&A, 437, 1159

Beck, C., Bellot Rubio, L. R., Schlichenmaier, R., et al. 2007, A\&A, 472, 607

Beck, C., Schmidt, W., Rezaei, R., et al. 2008, A\&A, 479, 213

Beck, C., Khomenko, E., Rezaei, R., et al. 2009, A\&A, 507, 453

Cabrera Solana, D., Bellot Rubio, L. R., \& del Toro Iniesta, J. C. 2005, A\&A, 439, 687

Carlsson, M., \& Stein, R. F. 1997, ApJ, 481, 500

Cavallini, F. 2006, Sol. Phys., 236, 415

Collados, M., Lagg, A., Díaz Garcí A, J. J., et al. 2007, in The Physics of Chromospheric Plasmas, ed. P. Heinzel, I. Dorotovič, \& R. J. Rutten, ASP Conf. Ser., 368, 611

Cram, L. 1978, A\&A, 70, 345

Freytag, B., Steffen, M., \& Dorch, B. 2002, Astron. Nachr., 323, 213

Gingerich, O., Noyes, R. W., Kalkofen, W., et al. 1971, Sol. Phys., 18, 347

Holweger, H., \& Mueller, E. A. 1974, Sol. Phys., 39, 19

Huenerth, G., \& Ulmschneider, P. 1995, A\&A, 293, 166

Liu, S.-Y. 1974, ApJ, 189, 359

Rammacher, W., \& Ulmschneider, P. 1992, A\&A, 253, 586

Rammacher, W., \& Ulmschneider, P. 2003, ApJ, 589, 988

Rezaei, R., Bruls, J. H. M. J., Schmidt, W., et al. 2008, A\&A, 484, 503

Ruiz Cobo, B. 1998, Ap\&SS, 263, 331

Ruiz Cobo, B., \& del Toro Iniesta, J. C. 1992, ApJ, 398, 375

Rutten, R. J., \& Uitenbroek, H. 1991, Sol. Phys., 134, 15

Rutten, R. J., de Wijn, A. G., \& Sütterlin, P. 2004, A\&A, 416, 333

Schaffenberger, W., Wedemeyer-Böhm, S., Steiner, O., et al. 2005, in Chromospheric and Coronal Magnetic Fields, ed. D. E. Innes, A. Lagg, \& S. A. Solanki, ESA SP, 596

Schaffenberger, W., Wedemeyer-Böhm, S., Steiner, O., et al. 2006, in Solar MHD Theory and Observations: A High Spatial Resolution Perspective, ed. J. Leibacher, R. F. Stein, \& H. Uitenbroek, ASP Conf. Ser., 354, 345 Ulmschneider, P. 1994, A\&A, 288, 1021

Wedemeyer, S., Freytag, B., Steffen, M., Ludwig, H.-G., \& Holweger, H. 2004, A\&A, 414, 1121 